\title{
Magnetization orientation dependence of the quasiparticle spectrum and hysteresis in ferromagnetic metal nanoparticles
}

\author{
A. Cehovin \\ Division of Solid State Theory, Department of Physics, \\ Lund University, SE-223 62 Lund, Sweden \\ C.M. Canali \\ Department of Technology, Kalmar University, \\ 39182 Kalmar, Sweden, and Division of Solid State Theory, \\ Department of Physics, Lund University, SE-223 62 Lund, Sweden \\ A.H. MacDonald \\ Department of Physics, University of Texas at Austin, Austin TX 78712
}

(Dated: November 2, 2018) 


\begin{abstract}
We use a microscopic Slater-Koster tight-binding model with short-range exchange and atomic spin-orbit interactions that realistically captures generic features of ferromagnetic metal nanoparticles to address the mesoscopic physics of magnetocrystalline anisotropy and hysteresis in nanoparticle quasiparticle excitation spectra. Our analysis is based on qualitative arguments supported by self-consistent Hartree-Fock calculations for nanoparticles containing up to 260 atoms. Calculations of the total energy as a function of magnetization direction demonstrate that the magnetic anisotropy per atom fluctuates by several percents when the number of electrons in the particle changes by one, even for the largest particles we consider. Contributions of individual orbitals to the magnetic anisotropy are characterized by a broad distribution with a mean more than two orders of magnitude smaller than its variance and with no detectable correlations between anisotropy contribution and quasiparticle energy. We find that the discrete quasiparticle excitation spectrum of a nanoparticle displays a complex non-monotonic dependence on an external magnetic field, with abrupt jumps when the magnetization direction is reversed by the field, explaining recent spectroscopic studies of magnetic nanoparticles. Our results suggests the existence of a broad cross-over from a weak spin-orbit coupling to a strong spin-orbit coupling regime, occurring over the range from approximately 200- to 1000-atom nanoparticles.
\end{abstract}




\section{INTRODUCTION}

Interest in the properties of magnetic nanoparticles has grown recently, partly because of advances in synthesis and measurement techniques and partly because of potential applications for high storage-density magnetic media and spin electronics. Ferromagnetic nanoparticles with diameters of a few nanometers containing of order of 1000 or fewer atoms can now be reliably fabricated and studied with a variety of different methods [1, 2, 3]. Small monodomain magnetic particles have traditionally [4, 5] been described using classical $m i$ cromagnetic theory, in which the total energy is expressed as a function of the magnetization orientation. Shape and magnetocrystalline magnetic anisotropy leads to a dependence of energy on orientation, to barriers that separate minima that occur at easy magnetization orientations [6], and to hysteretic discontinuous changes in orientation as a function of the strength of an external magnetic field. When the size of a magnetic particle is only a few nanometers, the discrete nature of its quantum energy spectrum can be directly observable at low temperatures and starts to affect the magnetic properties of the particle.

A milestone in the experimental study of individual ferromagnetic metal nanoparticles was achieved recently by Deshmukh, Guéron, Ralph et.al.[1, 7], using single-electron tunneling spectroscopy. By exploiting the Coulomb blockade effect, these experimentalists were able to resolve individual quantum states in the discrete many-body excitation spectrum of single ferromagnetic metal nanograins with sizes in the range from 1 to $4 \mathrm{~nm}$. As in bulk ferromagnetic metals, the low-energy excitations of the nanoparticles that were probed in these experiments involve both particle-hole excitations of the electronic quasiparticles and quantized collective excitations of the magnetization-orientation field that appears in the classical micromagnetic theory. In an initial attempt to achieve an understanding of the novel physics evident in the external field dependence of the experimental excitation spectra, two of us recently [8] analyzed a simple quantum model with long range exchange interactions. We were able to demonstrate explicitly that the low-energy excitations of a ferromagnetic metal nanoparticle are specified by the occupation numbers of its quasiparticle

orbitals, as in a Fermi liquid, and by the global orientation of the aligned spins of all singleoccupied (and therefore spin-polarized) orbitals. This model is not, however, able to account realistically for the influence of spin-orbit interactions, which play the essential role in controlling the complex hysteretic behavior seen in these experiments[1, [7. Kleff, von Delft, 
Deshmukh and Ralph [9, 10 have proposed that the single-electron tunneling spectroscopy experiments can be explained by accounting for non-equilibrium spin accumulation, and by assuming that the magnetic anisotropy energy of a small magnetic particle has surprisingly large fluctuations as a function of the number of electrons on the particle. This assumption leads to a non-trivial magnetic field dependence of tunneling resonances that resembles experimental behavior. More critically, these authors point out that if non-equilibrium spin and quasi-particle excitations both occur [10, the low energy spectra are characterized by many closely spaced resonances, consistent with experiment [10]. In Ref. 11 we presented a possible approach toward achieving a unified and consistent quantum description of both collective and quasiparticle physics in magnetic nanoparticles.

The attempts put forward in Refs. 8, 9, 10 to develop a quantum description of ferromagnetic metal nanoparticles do not address the microscopic origin of the magnetic anisotropy and the fluctuations of this quantity that are a necessary consequence of spin-orbit interactions in mesoscopic systems. This article addresses these issues and, more in general, investigates the changes in magnetic properties of small metallic nanoparticles that occur because of the finite spacing between quasiparticle levels near the Fermi energy. Our conclusions follow from qualitative arguments based on perturbation theory expressions for the influence of spin-orbit interactions on quasiparticle energy levels, and on numerical studies of a simplified model that we believe is sufficiently realistic to describe generic aspects of the interrelated mesoscopic physics of quasiparticle energy-levels and magnetic anisotropy energy in ferromagnetic metal nanoparticles. We are particularly interested in the variation of quasiparticle energies with fields on the scale of the coercive field, which is trivial in the absence of spin-orbit interactions, but entirely non-trivial in their presence. The model we study is based on a Slater-Koster tight binding Hamiltonian and a mean-field treatment of exchange interactions. Our aim is to understand the dependence of magnetic anisotropy and quasiparticle energy-level-spacing statistics on particle size and shape, external magnetic field, and, with single-electron-transistor systems in mind, also on electron number. We find that, because of the absence of strong correlations between angular momentum operator matrix elements and orbital energy differences, the size of spin-orbit induced energy shifts in nanoparticles and bulk perfect crystals are similar. On the basis of estimates for energy shifts and for avoided crossing gaps, we predict that a crossover from weak to strong quasiparticle spin-orbit scattering will occur over the range from approximately 200-atom 
to approximately 1000-atom nanoparticles. We find that for small particles the contribution of individual quasiparticle orbitals to the magnetocrystalline anisotropy energy has a wide distribution, characterized by a variance comparable to the spin-orbit-scattering lifetime broadening energy, $\hbar \tau_{S O}^{-1}$, and a mean that is smaller by more than two orders of magnitude. Surprisingly, we find no measurable correlation between the contributions to magnetic anisotropy from quasiparticles that are close in energy in this limit. As a result of the statistical properties of the quasiparticle magnetic anisotropies, the total magnetic anisotropy per atom fluctuates by several percents when the number of electrons in the nanoparticle changes by one, even for particles containing 260 atoms. Finally, in agreement with experiment [1, 7], we find that the quasiparticle excitation spectrum exhibits a complex non-monotonic behavior as a function of an external magnetic field, with abrupt jumps when the magnetization orientation of a nanoparticle changes discontinuously in response to the field. Our analysis provides insight into mesoscopic fluctuations of the micromagnetic energy functional which appears in the classical theory of a magnetic nanoparticle.

Our paper is organized in the following way. In Section II we introduce the model and describe the formalism. In Section [III we analyze the qualitative change in quasiparticle energy-level statistics induced by spin-orbit interactions, and discuss the connection between the quasiparticle properties and the magnetic anisotropy of a ferromagnetic nanoparticle. Fluctuations of the magnetic anisotropy as a function of spin-splitting field, atom number, and electron number are investigated in Section [D. Magnetic hysteresis and the external field dependence of quasi-particle energy levels are discussed in Section $\mathrm{V}$. Finally, in Section V1 we summarize our findings and present our conclusions.

\section{THE MODEL}

We model the nano-particle as a cluster of $\mathcal{N}_{a}$ atoms located on the sites of a truncated crystal. The numerical results we present here are for a cobalt cluster whose truncated f.c.c. crystal is circumscribed by a hemisphere whose equator lies in the $x y$-plane of the f.c.c. crystal 37. 


\section{A. Tight-binding Hamiltonian and Slater-Koster parameters}

The model we use is intended to qualitatively capture the physics of a transition-metal, itinerant-electron ferromagnet. We use a s-p-d tight-binding model for the quasiparticle orbitals, with 18 orbitals per atom including the spin-degree of freedom. Nine orbitals per Co atom are occupied for in neutral nanoparticles. The full Hamiltonian is,

$$
\mathcal{H}=\mathcal{H}_{\text {band }}+\mathcal{H}_{\text {exch }}+\mathcal{H}_{\text {SO }}+\mathcal{H}_{\text {Zee }} .
$$

Here $\mathcal{H}_{\text {band }}$ is a one-body term describing the orbital motion of the electrons. In second quantization, it has the form

$$
\mathcal{H}_{\text {band }}=\sum_{i, j} \sum_{s} \sum_{\mu_{1}, \mu_{2}} t_{\mu_{1}, \mu_{2}, s}^{i, j} c_{i, \mu_{1}, s}^{\dagger} c_{j, \mu_{2}, s}
$$

where $c^{\dagger}$ and $c$ are Fermion creation and annihilation operators for single-particle states labeled by $i, \mu, s$. The indices $i, j$ are atomic site labels, and $t^{i, j}$ couples up to second nearestneighbors. The indices $\mu_{1}, \mu_{2}$ label the nine distinct atomic orbitals (one $4 s$, three $4 p$ and five $3 d$ ). The spin degrees of freedom, labeled by the index $s$, double the number of orbitals at each site. It is useful for us to vary the spin-quantization axis, which is specified by a unit vector $\hat{\Omega}(\Theta, \Phi)$ where $\Theta$ and $\Phi$ are the usual angular coordinates defined with respect to the f.c.c. crystal axes. The parameters $t_{\mu_{1}, \mu_{2}, s}^{i, j}$ are Slater-Koster parameters 12 obtained after performing a Löwdin symmetric orthogonalization procedure [13] on the set of Slater-Koster parameters for non-orthogonal atomic orbitals of bulk spin-unpolarized Co [14.

The electron-electron interaction term in Eq. (1) is simplified by introducing explicitly [38] only the ferromagnetic exchange interaction, $\mathcal{H}_{\text {exch }}$, between the electrons spins of $d$ orbitals on the same atomic site. These interactions are largely responsible for magnetic order in transition metal ferromagnets:

$$
\mathcal{H}_{\text {exch }}=-2 U_{d d} \sum_{i} \vec{S}_{d, i} \cdot \vec{S}_{d, i}
$$

where

$$
\vec{S}_{d, i}=\sum_{\mu \in d} \vec{S}_{i, \mu}=\sum_{\mu \in d} \frac{1}{2} \sum_{s, s^{\prime}} c_{i, \mu, s}^{\dagger} \vec{\tau}_{s, s^{\prime}} c_{i, \mu, s^{\prime}}
$$

The parameter $U_{d d}$ in Eq. (3) determines the strength of the exchange interaction and is set equal to $1 \mathrm{eV}$. 
This value of $U_{d d}$ gives rise in our finite clusters to an average magnetic moment per atom of the order of $2 \mu_{\mathrm{B}}$, which is larger than the bulk value in Co 14, in agreement with other calculations [15] and experimental results [16, 17] for Co clusters. This value of $U_{d d}$ is also approximately consistent with a mean-field relationship, derived below, between band spin-splitting and magnetization, using bulk values [14] for these two quantities. In Eq. (四), $\vec{\tau}$ is a vector whose components $\tau^{\alpha}, \alpha=x, y, z$ are the three Pauli matrices.

The third term in Eq. (四), $\mathcal{H}_{\mathrm{SO}}$, is a one-body operator, essentially atomic in character, representing the spin-orbit interaction. It can be written as 18

$$
\mathcal{H}_{\mathrm{SO}}=\xi_{d} \sum_{i} \sum_{\mu, \mu^{\prime}, s, s^{\prime}}\left\langle\mu, s|\vec{L} \cdot \vec{S}| \mu^{\prime}, s^{\prime}\right\rangle c_{i, \mu, s}^{\dagger} c_{i, \mu^{\prime}, s^{\prime}}
$$

The atomic matrix elements $\left\langle\mu, s|\vec{L} \cdot \vec{S}| \mu^{\prime}, s^{\prime}\right\rangle \equiv\left\langle i, \mu, s|\vec{L} \cdot \vec{S}| i, \mu^{\prime}, s^{\prime}\right\rangle$ can be been calculated explicitly as a function of the direction of the magnetization $\hat{\Omega}[19]$. The energy scale $\xi_{d}$, which characterizes the coupling between spin and orbital degrees of freedom, varies in the range from 50 to $100 \mathrm{meV}$ in bulk 3d transition metal ferromagnets[20]. For our calculations we have used $\xi_{d}=82 \mathrm{meV}$, taken from Ref. [21].

Spin-orbit coupling gives rise to a dependence of the total energy of a ferromagnet on the direction of its spontaneous magnetization, an effect known as magnetocrystalline anisotropy [39]; with spin-orbit coupling the magnetization is partially orbital in character [22, 23] and is sensitive to orbital anisotropy due to crystal-field interactions on an atomic site, due to the spatial arrangement of the atoms neighboring that site to which electrons can hop, and due to the overall shape of the full nanoparticle that becomes available to an electron after many hops [40]. We will see below that the hemispherical shape of the nanoparticles we have studied plays the dominant role in determining their magnetic anisotropy energy.

The last term in Eq. (11) is a local one-body operator, representing the Zeeman coupling of the orbital and spin degrees of freedom to an external magnetic field $\vec{H}_{\text {ext }}$ :

$$
\begin{aligned}
\mathcal{H}_{\mathrm{Zee}} & =-\mu_{\mathrm{B}} \sum_{i} \sum_{\mu, \mu^{\prime}, s, s^{\prime}}\left\langle\mu, s \mid\left(\vec{L}+g_{s} \vec{S} \mid \mu^{\prime}, s^{\prime}\right)\right\rangle \cdot \vec{H}_{\mathrm{ext}} c_{i, \mu, s}^{\dagger} c_{i, \mu^{\prime}, s^{\prime}} \\
& \left.=-\mu_{\mathrm{B}} \sum_{i} \vec{H}_{\mathrm{ext}} \cdot\left\{\sum_{\mu, \mu^{\prime}, s}\left\langle\mu, s|\vec{L}| \mu^{\prime}, s\right)\right\rangle c_{i, \mu, s}^{\dagger} c_{i, \mu^{\prime}, s}+\frac{g_{s}}{2} \sum_{\mu, s, s^{\prime}} c_{i, \mu, s}^{\dagger} \vec{\tau}_{s, s^{\prime}} c_{i, \mu, s^{\prime}}\right\} .
\end{aligned}
$$

The extreme sensitivity of magnetization orientation to external magnetic field is a combined effect of the collective behavior of many electrons in a ferromagnetic nanoparticle and 
the smallness of the magnetic anisotropy energy relative to the overall energy gain associated with ferromagnetic order. The most delicate physics of a ferromagnetic nanoparticle, and in our view the most interesting, is that associated with magnetization direction reorientation by weak external magnetic fields. Thus the interplay between the Zeeman term and the magnetic anisotropy produced by spin-orbit interactions is at the heart of the physics we intend to address.

\section{B. Mean-field approximation}

We seek a ferromagnetic solution to the mean-field equations for this model, decoupling the quartic term in the exchange interaction using the ansatz

$$
\vec{S}_{d, i}=\left\langle\vec{S}_{d, i}\right\rangle+\delta \vec{S}_{d, i}
$$

ignoring terms that are second order in $\delta \vec{S}_{d, i}$, and determining ground state expectation values, $\langle\ldots\rangle$, self-consistently. This standard procedure leads to a Hamiltonian that can be diagonalized numerically and to a self-consistency condition that can be solved iteratively to determine the mean-field order parameters. For the present calculation we have simplified this procedure further, by averaging the spin-splitting exchange mean field,

$$
\vec{h}_{i} \equiv h \hat{\Omega}=\frac{U_{d d}}{g_{s} \mu_{B}} 2\left\langle\vec{S}_{d, i}\right\rangle
$$

over all sites. Our motivation for doing so is to simplify the magnetic anisotropy energy landscape discussed below, forcing all spins to change their orientations coherently. We recognize that complicated non-collinear spin configurations 24, 25 commonly occur in magnetic nanoparticles, and that under the action of an external field small groups of atoms can change their orientation relative to other parts of the nanoparticle. Complex magnetization reorientation processes are obvious in addition spectroscopy experiments [7]. By forcing atoms to change their magnetic orientations coherently, we are restricting our attention to relatively large nanoparticles $\left(\mathcal{N}_{a}>50\right)$ in which most atoms have parallel spins and to nanoparticles with simple bistable hysteretic behavior in which the physics we address will be easier to study. The beautiful series of detailed SQUID magnetometry experiments by Wernsdorfer and colleagues [3, 26] demonstrate that nanoparticles can be prepared that do have simple coherent magnetization reversal properties. 
By using a simplified model Hamiltonian we are able to deal with larger nanoparticle systems than would be possible with a first principles calculation [15, 21, 24, 27]; since we are interested only in generic aspects of the ferromagnetic nanoparticle physics there is little to gain from the additional realism that could be achieved by performing self-consistent spin-density functional calculations.

Given the averaged spin-splitting field $\vec{h}$, the mean-field Hamiltonian is now a single-body operator

$$
\mathcal{H}_{\mathrm{MF}}(\vec{h})=\mathcal{H}_{\text {band }}+\mathcal{H}_{\mathrm{SO}}+\mathcal{H}_{\text {Zee }}+\frac{\vec{h} \cdot \vec{h}}{2 U_{d d}}\left(g_{s} \mu_{\mathrm{B}}\right)^{2} N_{a}-g_{s} \mu_{\mathrm{B}} \vec{h} \cdot \sum_{i} 2 \vec{S}_{d, i}
$$

The self-consistent spin-spitting field is also the field, denoted by $\vec{h}^{\star}$ below, at which the total ground-state energy function $E(\vec{h})=\left\langle\mathcal{H}_{\mathrm{MF}}(\vec{h})\right\rangle$ is minimized. Notice that $g_{s} \mu_{\mathrm{B}} h^{\star}$ has the dimension of an energy. In fact, in absence of spin-orbit interaction, $2 g_{s} \mu_{\mathrm{B}} h^{\star}$ can be identified with the band spin-splitting $\Delta=\epsilon_{\mathrm{F} a}^{0}-\epsilon_{\mathrm{F} i}^{0}$, where $\epsilon_{\mathrm{F} a}^{0}$ and $\epsilon_{\mathrm{F} i}^{0}$ are the majority and minority spin quasi-particle Fermi energies. [41] Diagonalization of the quadratic Hamiltonian yields

a set of quasi-particle energies $\left\{\epsilon_{n, s}\right\}, n=1,2, \ldots$, which in the ground state are filled up to a Fermi energy determined by the number of valence electrons in the nanoparticle. Strictly speaking, because of the spin-orbit interaction, the spin character of the corresponding eigenstates $\left|\psi_{n, s}\right\rangle$ is not well defined.

For small nanoparticles, most eigenstates have predominantly spin-up or spin-down character and we sometimes use this property to assign spin labels $s=\uparrow$ for spins along the order direction and $s=\downarrow$ for reversed spins.

\section{SPIN-ORBIT INTERACTIONS AND THE MAGNETIC ANISOTROPY EN- ERGY}

The magnetic anisotropy energy of small ferromagnetic particles [3] has two fundamentally distinct origins, long-range magnetic-dipole interactions which cause a dependence on overall sample shape and short-range exchange interactions that, because of atomic-like spin-orbit interactions, are sensitive to all aspects of the electron hopping network including bulk crystal symmetry, facet orientations, and also overall sample shape. We concentrate here on spin-orbit-induced magnetocrystalline anisotropy which gives rise to the most interesting physics in ferromagnetic nanoparticles. When magnetostatic shape anisotropy is important, 
it can be added as a separate contribution. We begin our discussion with some qualitative estimates of the effect of spin-orbit interactions that are based on perturbation theory [28, 22].

\section{A. Perturbation theory considerations}

In bulk 3d transition metal ferromagnets, spin-orbit interactions are relatively weak. Their coupling strength is less than $10 \%$ of the d-band width $W_{d}$ in bulk materials [20], allowing the energy shifts they produce to be estimated perturbatively. Because of angular momentum quenching in the absence of external fields, the expectation value of $H_{\mathrm{SO}}$ is zero, even in case of ferromagnets[2].

The quasi-particle energy shift due to spin-orbit interactions is given by second-order perturbation theory as

$$
\epsilon_{\mathrm{SO}} \equiv \epsilon_{n, s}-\epsilon_{n, s}^{0}=\frac{\left(\xi_{d}\right)^{2}}{4} \sum_{\substack{s^{\prime} \\ m \neq n}} \frac{\left|\left\langle\psi_{m, s^{\prime}}^{0}|\vec{L}| \psi_{n, s}^{0}\right\rangle \cdot \vec{\tau}_{s^{\prime}, s}\right|^{2}}{\epsilon_{n, s}^{0}-\epsilon_{m, s^{\prime}}^{0}},
$$

where $\left|\psi_{n, s}^{0}\right\rangle$ and $\epsilon_{n, s}^{0}$ are respectively the single-particle eigenstates and energies in the absence of spin-orbit interaction. In small particles, the importance of the spin-orbit interactions can be assessed by comparing the spin-orbit energy shift $\epsilon_{\mathrm{SO}}$ with the single-particle mean-level spacing $\delta$.

In an infinite periodic solid only states at the same $\vec{k}$ are coupled and these are separated energetically by an energy comparable to the bandwidth $W_{d}$. In a nanoparticle a given state will be coupled to many other orbitals, but the coupling matrix elements will be reduced in accord with the following sum rule:

$$
\sum_{\substack{s^{\prime} \\ m \neq n}}\left|\left\langle\psi_{m, s^{\prime}}^{0}|\vec{L}| \psi_{n, s}^{0}\right\rangle \cdot \vec{\tau}_{s^{\prime}, s}\right|^{2}=\left\langle\psi_{n, s}^{0}|\vec{L} \cdot \vec{L}| \psi_{n, s}^{0}\right\rangle \sim 4
$$

The estimate for the right hand side of Eq. 11 is based on the atomic character of the angular momentum in our model, and uses that $\vec{L} \cdot \vec{L} \sim[5 * 6+3 * 2+1 * 0] / 9$, with the estimate of the typical $\vec{L} \cdot \vec{L}$ expectation value representing an average over $d, s$ and $p$ orbitals. It follows that, unless there are important correlations between angular momentum matrix elements and quasiparticle energy differences, the typical shift in energy caused by the spin-orbit interaction is $\epsilon_{\mathrm{SO}} \sim \frac{\left(\xi_{d}\right)^{2}}{W_{d}}$, in both bulk crystals and in nanoparticles, 
which is in the range between 1 to $10 \mathrm{meV}$. For example in Co, using $\xi_{d}=82 \mathrm{meV}$ and $W_{d} \sim 5 \mathrm{eV}$, this rough estimate gives for the magnitude of the spin-orbit energy shift $\epsilon_{\mathrm{SO}}$ $\sim 1.3 \mathrm{meV}$. The sign of the shift might be expected to be sensitive to the spacing of nearby quasiparticle orbitals. The anisotropy energy, that is, the dependence of the total band energy on the magnetization orientation, is given to a good approximation by a partial canceling sum of spin-orbit induced energy shift dependences on magnetic orientation.

In the approximation that the exchange field is orbital independent, majority spin and minority spin orbitals are identical and differ only in their occupation numbers. In this approximation there is no contribution to the anisotropy energy from doubly occupied orbitals. Because of the cancellations, the anisotropy energy per atom is much smaller than $\epsilon_{\mathrm{SO}}$. For example, the zero-temperature anisotropy energy per atom in bulk is $60 \mu \mathrm{eV}$ for h.c.p. Co, and $\approx 1 \mu \mathrm{eV}$ for b.c.c. Fe and f.c.c. $\mathrm{Ni}$ 30]. In a finite or disordered system, there will always be perturbative coupling to quasiparticle states close in energy in Eq. 10, but the matrix elements, which satisfy the sum rule of Eq. 11, will be distributed among many states and typical energy shifts in nanoparticles should generally be comparable to those in bulk perfect crystals. Typically net anisotropy energies per atom in small magnetic particles are larger than the bulk because of the loss of symmetry at the surface.

An important quantity used to characterize the strength of spin-orbit interactions in bulk systems and large nanoparticles is the the spin-orbit scattering time, $\tau_{\mathrm{SO}}[31,32,33,34$.

In the weak coupling regime, it is given by the Fermi's golden rule

$$
\hbar \tau_{\mathrm{SO}}^{-1}=\frac{\left(\xi_{d}\right)^{2}}{4} \sum_{\substack{s^{\prime} \\ m \neq n}}\left|\left\langle\psi_{m, s^{\prime}}^{0}|\vec{L}| \psi_{n, s}^{0}\right\rangle \cdot \vec{\tau}_{s^{\prime}, s}\right|^{2} \delta\left[\epsilon_{n, s}^{0}-\epsilon_{m, s^{\prime}}^{0}\right],
$$

where the $\delta$-function is understood to be broadened to a width much larger than the level spacing. Assuming that there is no correlation between angular momentum matrix elements and orbital energy differences, it follows from the sum rule mentioned above that

$$
\hbar \tau_{\mathrm{SO}}^{-1} \sim \epsilon_{\mathrm{SO}} \sim \frac{\xi_{d}^{2}}{W_{d}}
$$

The absence of strong correlations between energy differences and angular momentum matrix elements, a property that we find somewhat surprising, has been verified numerically as we discuss below. The character of the nanoparticle quasiparticle energy spectrum changes when these intensive energy scales become comparable to the nanoparticle level spacing $\delta$. 


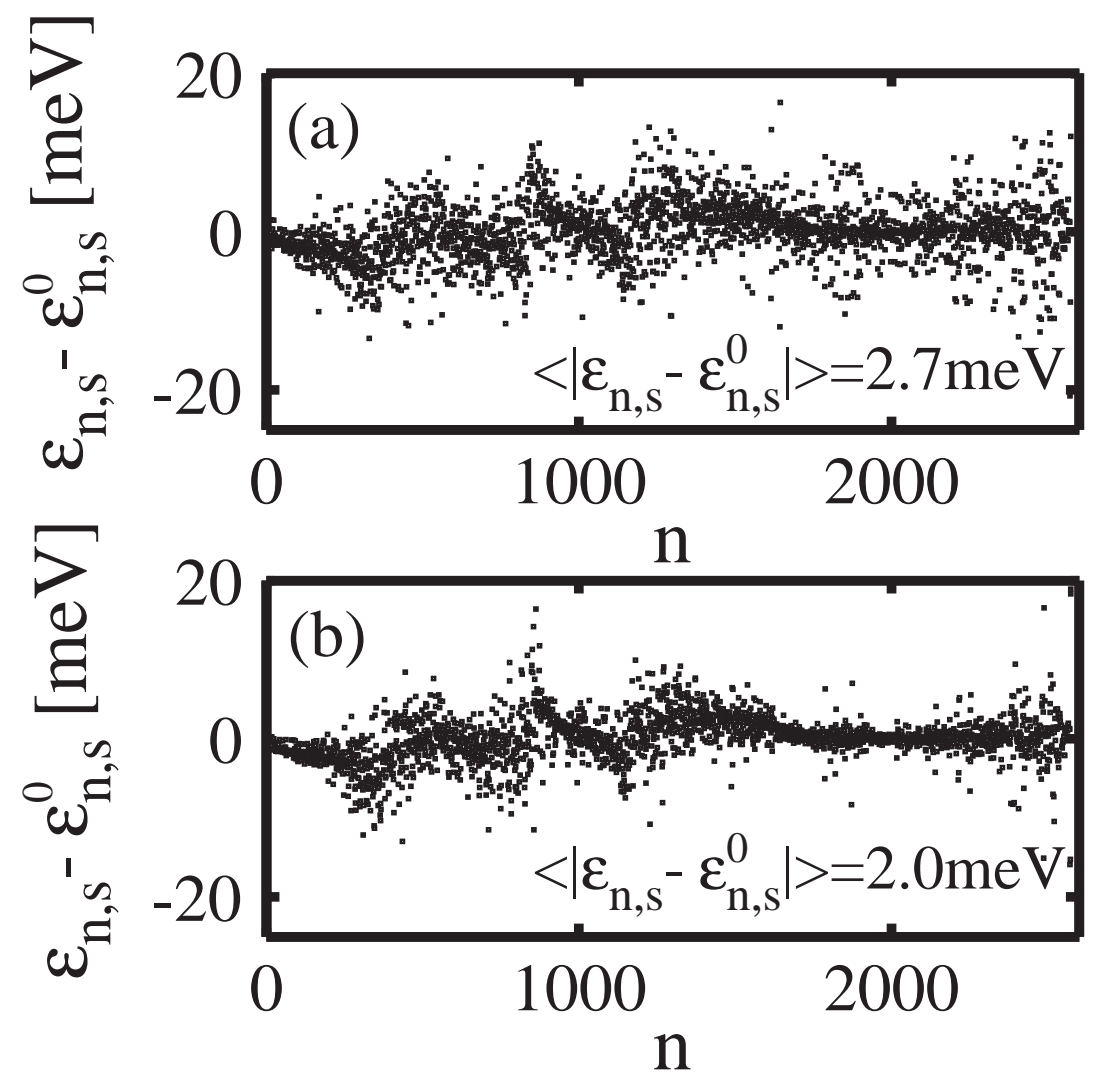

FIG. 1: Single-particle energy shifts caused by spin-orbit interactions in a ferromagnetic Cobalt nanoparticle of 143 atoms. The single-particle mean level spacing at the Fermi energy is $\delta \approx 4.3$ meV. (a) Magnetization in the z-direction. (b) Magnetization in the x-direction.

\section{B. Numerical Results for a Co nanoparticle}

The qualitative considerations of the previous section provide a framework for thinking about the effects of spin-orbit interactions. Our microscopic model, on the other hand, allows us to explore realistic magnetic nanoparticle systems in great detail. We have studied numerically nanoparticles containing up to 260 atoms. Most of the results presented below are for hemispherical 143-atom nanoparticles. The calculations have been performed with a R12000 300MHz processor on an SGI Origin 2000 computer. Diagonalizations rely on LAPACK drivers. A single diagonalization of the Hamiltonian for a 143-atom cluster has a running time of approximately 1 hour, and requires around $750 \mathrm{Mb}$ of internal memory. In Fig. [1] we plot the energy shifts caused by spin-orbit interaction for a hemispherical Cobalt nanoparticle of 143 atoms with a f.c.c. crystal structure. For this nanoparticle size, the 

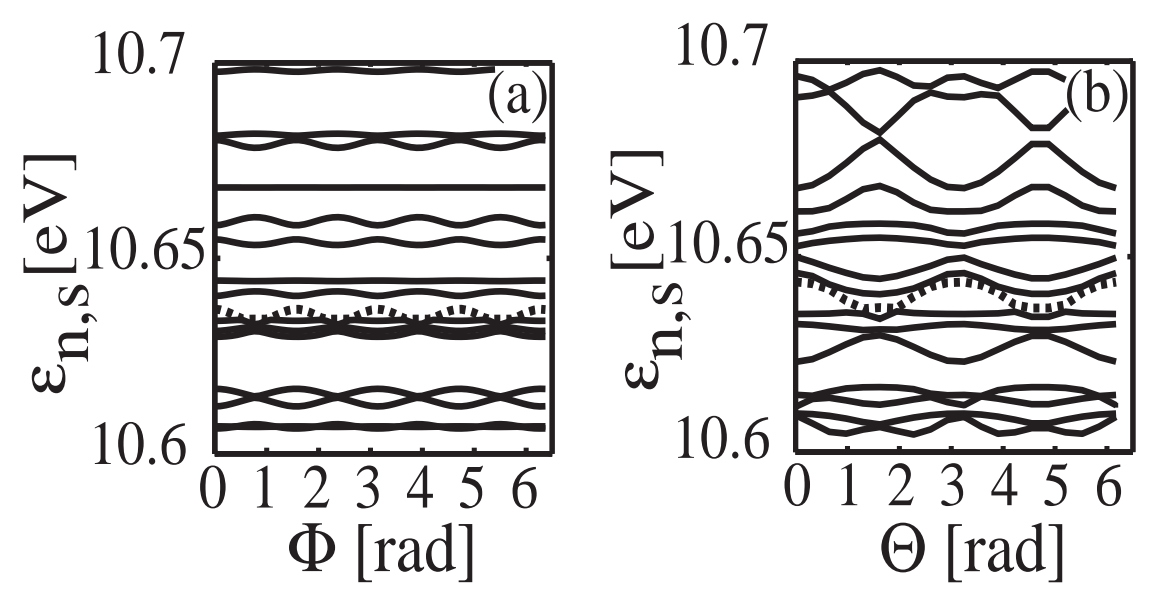

FIG. 2: Variation of a few quasiparticle energies of a 143-atom Co nanoparticle as a function of the direction of the magnetization $\hat{\Omega}$. The Fermi level is the dotted line. (a) $\hat{\Omega}$ lies in the $x y$-plane and $\Phi$ is the angle with $x$-axis. (b) $\hat{\Omega}$ lies in the $z x$-plane and $\Theta$ is the angle with the $z$-axis.

minority and majority single-particle mean-level spacings at the Fermi level are $\delta_{\downarrow} \approx 4.9$ $\mathrm{meV}$ and $\delta_{\uparrow} \approx 50 \mathrm{meV}$ respectively, when spin-orbit interactions are absent. The singleparticle mean-level spacing averaged over all states (i.e. without distinguishing between majority and minority levels) is $\delta \approx 4.3 \mathrm{meV}$ at the Fermi level 42 . The spin-orbit induced shifts are both positive and negative and their absolute values go from $1 \mathrm{meV}$ up to $10 \mathrm{meV}$. The average absolute value of the energy shifts is $2.7 \mathrm{meV}$ when the magnetization is in the z-direction, and $2 \mathrm{meV}$ when the magnetization is in the $x y$-plane, consistent with the rough estimates above. We also note that there is not a strong correlation between the sign of the energy shift and the energy of the orbital.

Because of the spin-orbit interaction, each individual eigenlevel has an energy dependence on the spin-splitting field (or magnetization) direction $\hat{\Omega}$. To illustrate typical properties of these dependences, we plot in Fig. [2] the variation of a few energy levels around the Fermi level for cases in which the magnetization rotates in $z x$-plane and $x y$-planes respectively. In the absence of spin-orbit interactions there would be no dependence of any of these orbital energies on magnetization orientation.

Notice that the angle dependence in the $x y$-plane is considerably weaker than in the $z x$-plane, for the hemispherical nanoparticle we consider. This trend indicates that for our nanoparticles, it is the overall shape which dominates the spin-orbit induced anisotropy physics. For this size cluster, there are many narrowly avoided level crossings, a point to 

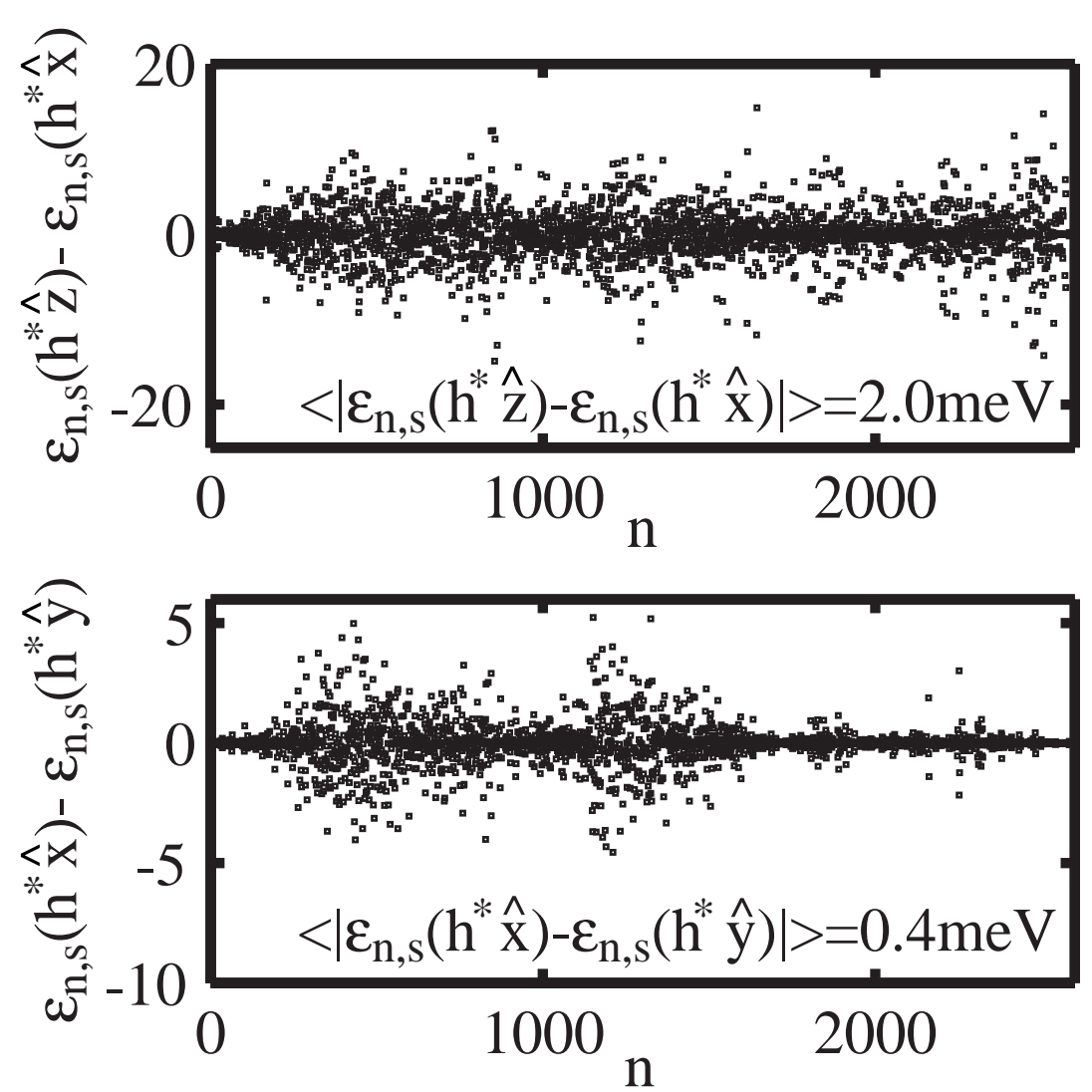

FIG. 3: Single-particle level anisotropies in the $x y$ - and $z x$-planes for a 143-atom nanoparticle.

which we return below. The difference in eigenvalue sensitivity to magnetization rotations in the two planes is clearly visible in Fig. [3], where we plot the single-particle level anisotropies $\epsilon_{n, s}\left(h_{\hat{z}}^{\star} \hat{z}\right)-\epsilon_{n, s}\left(h_{\hat{x}}^{\star} \hat{x}\right)$ and $\epsilon_{n, s}\left(h_{\hat{x}}^{\star} \hat{x}\right)-\epsilon_{n, s}\left(h_{\hat{y}}^{\star} \hat{y}\right)$ versus the eigenvalue index $n$. Here $h_{\hat{x}}^{\star}$ and $h_{\hat{z}}^{\star}$ are the magnitudes of the self-consistent spin-splitting field when its direction is along $\hat{x}$ and $\hat{z}$ respectively. We note that typical change in orbital energy between $\hat{x}$ and $\hat{z}$ direction magnetizations is only $\sim 30 \%$ smaller than the typical total shifts induced by spin-orbit interactions. On the other hand the typical difference in orbital energy between $\hat{x}$ and $\hat{y}$ direction magnetization is five times smaller than the corresponding spin-orbit induced energy shift. All orbital energies are relatively insensitive to the magnetization orientation within the $x y$-plane. In both cases the correlation between position within the band and the sign and magnitude of the energy shift is weak. In addition, energy shifts at nearby energies are weakly correlated. That is, the correlation function

$$
\left\langle\delta \epsilon_{n, s} \delta \epsilon_{n+k, s^{\prime}}\right\rangle-\left\langle\delta \epsilon_{n, s}\right\rangle^{2}, \quad \delta \epsilon_{n, s}=\epsilon_{n, s}\left(h_{1}^{\star} \hat{\Omega}_{1}\right)-\epsilon_{n, s}\left(h_{2}^{\star} \hat{\Omega}_{2}\right)
$$

where the average $\langle\ldots$.$\rangle is over the occupied levels n$, drops to zero very rapidly with $k$, as 


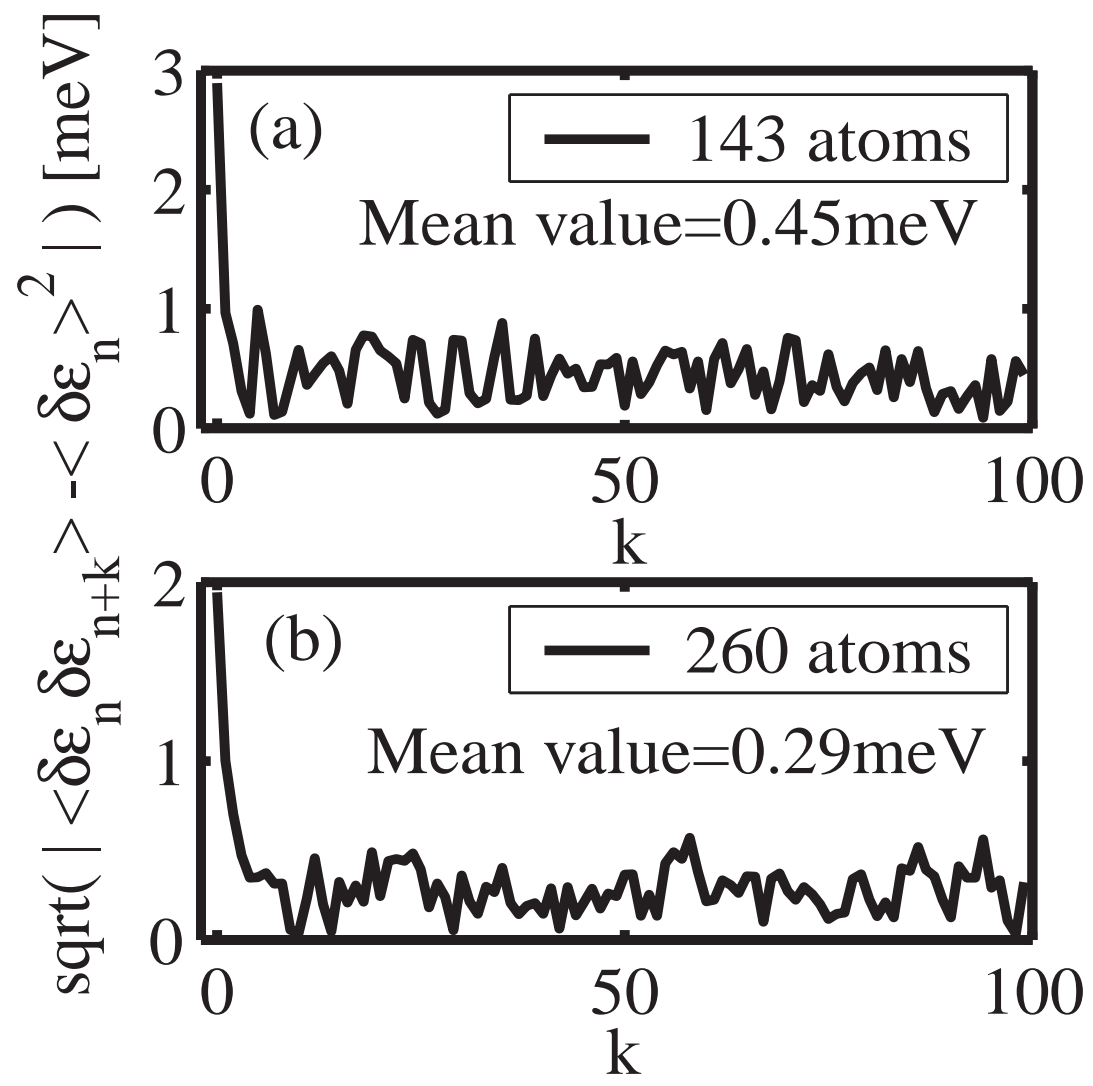

FIG. 4: Correlation function of single-particle anisotropies as defined in Eq. [14]. The correlation function drops immediately to zero for $k>0$. Note that the $k=0$ value of the correlation function is equal to the width of the anisotropy distribution, plotted in Fig. [5].

clearly shown in Fig. [1].

It is useful to consider the distribution of the quasiparticle anisotropies, $P\left(\delta \epsilon_{n, s}\right)$. As an example, in Fig. 5 we plot the distribution of anisotropies in the $z x$-plane, $\delta \epsilon_{n, s}=$ $\left[\epsilon_{n, s}\left(h^{\star} \hat{z}\right)-\epsilon_{n, s}\left(h^{\star} \hat{x}\right)\right]$, constructed with the $N=9 \times \mathcal{N}_{a}=9 \times 143$ occupied single-particle states of a 143-atom nanoparticle. The distribution has a width - characterized by the root mean square $-\Delta\left(\delta \epsilon_{n, s}\right) \sim 2.2 \xi_{d}^{2} / W_{d} \sim 2.9 \mathrm{meV}$ and a much smaller mean value $\left\langle\delta \epsilon_{n, s}\right\rangle=\left\langle\epsilon_{n, s}\left(h^{\star} \hat{z}\right)-\epsilon_{n, s}\left(h^{\star} \hat{x}\right)\right\rangle \sim 15 \mu \mathrm{eV}$. Note that $\Delta\left(\delta \epsilon_{n, s}\right)$ is exactly equal to the $k=0$ value of the correlation function displayed in Fig. [4]. Single particle anisotropies of groups of orbitals over within a specified energy range tend to be anti-correlated, leading to typical averages smaller than the variance of the distribution. The large difference between the distribution mean and variance will play an important role in Sec. IVB, where we discuss fluctuations of the anisotropy energy as a function of electron number. 


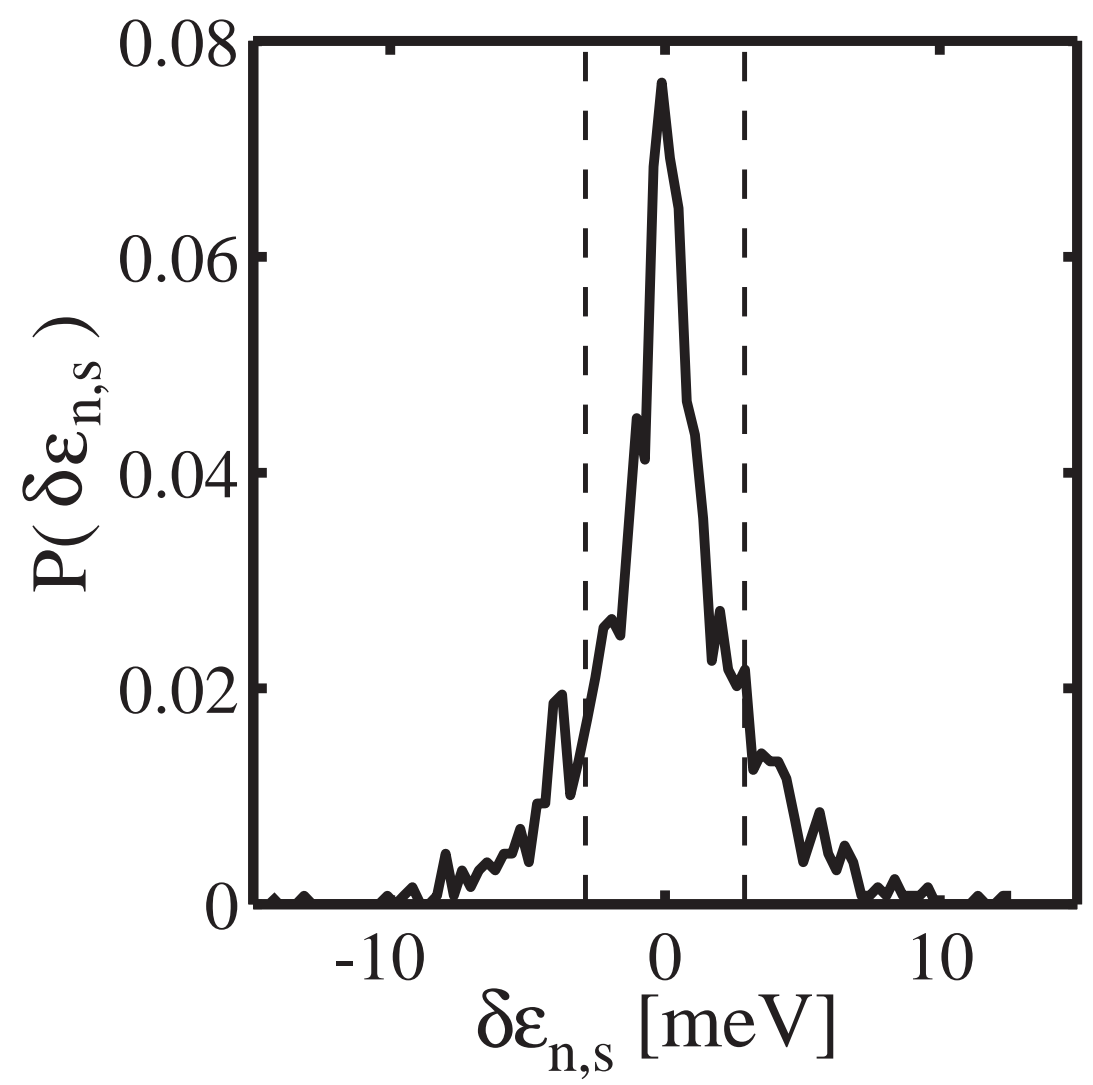

FIG. 5: Distribution function of single-particle anisotropies in the $z x$-plane, $\delta \epsilon_{n, s}=\left[\epsilon_{n, s}\left(h^{\star} \hat{z}\right)-\right.$ $\left.\epsilon_{n, s}\left(h^{\star} \hat{x}\right)\right]$, for a 143 -atom nanoparticle. The mean value is $\left\langle\delta \epsilon_{n, s}\right\rangle=15 \mu \mathrm{eV}$, the width of the distribution (enclosed by the vertical dashed lines) is $\Delta\left(\delta \epsilon_{n, s}\right)=2.9 \mathrm{meV}$.

The width of the distribution, $\Delta\left(\delta \epsilon_{n, s}\right)$, gives a measure of the average magnitude of the single-particle level anisotropy, and the ratio $\Delta\left(\delta \epsilon_{n, s}\right) / \delta$ characterizes the strength of mixing between quasiparticle orbitals that results from spin-orbit interactions. As mentioned previously, this identification of weak and strong spin-orbit interaction regimes is equivalent to the usual one 31, 32, 33, 34 based on the comparison of the spin-orbit scattering time $\tau_{\mathrm{SO}}$ and the mean-level spacing $\delta$.

When $\delta \tau_{\mathrm{SO}} / \hbar>>1$, a limit achieved for small enough particle size for any value of $\xi_{d}$, spin-orbit coupling is a relatively weak effect and there is little mixing between spin-up and spin-down states. As a consequence, the level crossings between states of predominantly opposite spins that occur as a function of the magnitude and orientation of $\vec{h}$, will be only weakly avoided. With increasing particle size, $\delta$ decreases and we enter the regime of strong-spin orbit interaction and strong level repulsion. The single-particle spectrum 


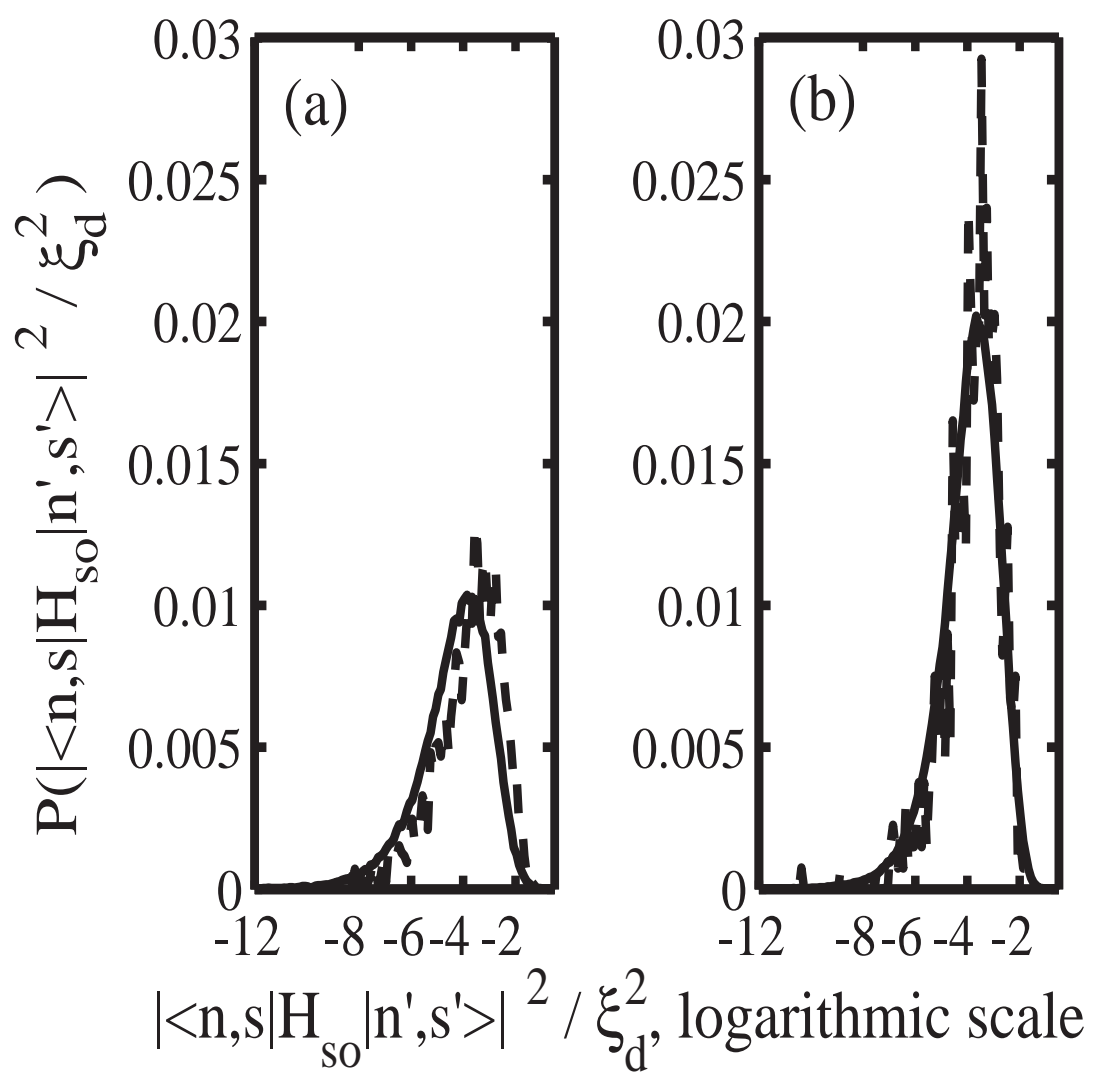

FIG. 6: Distribution function of spin-orbit matrix elements for a spin-polarized 143-atom nanoparticle. $|n, s\rangle$ and $\left|n^{\prime}, s^{\prime}\right\rangle$ are single-particle levels in absence of spin-orbit interaction. (a) $|n, s\rangle$ and $\left|n^{\prime}, s\right\rangle$ have like-spins; (b) $|n, s\rangle$ and $|n,-s\rangle$ have opposite-spins. Solid and dotted lines are for the cases of nearby levels $\left(\left|\epsilon_{n, s}^{0}-\epsilon_{n^{\prime}, s^{\prime}}^{0}\right| / \delta<3\right)$ and any pair of levels respectively.

becomes relatively rigid, level crossing will be strongly avoided, and $\delta$ will limit the variation of individual levels as a function of the magnetization direction.

Within our model we have found that the cross-over between these two regimes is very broad, it starts for nanoparticles containing of order 200 atoms and, as we argue below, it will be completed when the nanoparticles contain approximately 1000 atom. For a 143-atom nanoparticle, we are already approaching the cross-over regime; we find $\Delta\left(\delta \epsilon_{n, s}\right) \sim 2.9 \mathrm{meV}$ while the single-particle mean-level spacing at the Fermi level is $\delta \approx 4.3 \mathrm{meV}$. In this regime level crossings will be moderately avoided.

The typical size of avoided crossing gaps between opposite spin orbitals in small nanoparticles can be understood by the following argument. We first note that the unperturbed 
orbitals satisfy the following sum rule

$$
\left.\sum_{n^{\prime}}\left|\left\langle n^{\prime}, \downarrow|\vec{L} \cdot \vec{S}| n, \uparrow\right\rangle\right|^{2}=\left\langle n\left|L_{-} L_{+}\right| n\right\rangle\right) / 4 \sim 2 / 3
$$

The estimate for the right hand side of Eq. 15 is based on the on the same considerations leading to Eq. 11, and uses that $L_{-} L_{+} \sim 2 / 3 \vec{L} \cdot \vec{L} \sim 8 / 3$. If angular momentum matrix elements between orbitals are not correlated with the energy differences between these orbitals, and the matrix elements are reasonably narrowly distributed, this sum rule can be used to estimate the typical matrix element. Expectation values of the angular momentum operators are zero because of angular momentum quenching, and a finite fraction of the matrix elements vanish because of symmetries present in our rather regularly shaped nanoparticles. Aside from these features, we find numerically that correlations between matrix elements and energy differences are too small to be clearly observable. Fig. 6 shows the distribution function we have obtained for matrix elements between opposite and like-spin states. We have considered both the matrix element distribution for closely spaced levels and the distribution for any pair of levels, not necessarily nearby. The distributions are found to be very similar. Approximately $50 \%$ and $70 \%$ of matrix elements are zero for opposite-spin and like-spin cases respectively.

Based on these numerical results and the sum rule Eq. 15, we estimate the typical value of $\left|\left\langle n^{\prime}, \downarrow\left|H_{\mathrm{SO}}\right| n, \uparrow\right\rangle\right|^{2}$ as $\frac{2}{3} \xi_{d}^{2}$ divided by half the total number of s, p and d orbitals, $9 \mathcal{N}_{a} / 2$. This implies a typical non-zero matrix element equal to $\sim \xi_{d} \sqrt{.14 / \mathcal{N}_{a}}$.

In Fig. 7 we plot the average square matrix element vs. energy difference for a 143 atom cluster, obtaining remarkably precise agreement with this estimate provided that the energy differences are much smaller than the $d$-band width.

The average square matrix element for like spin orbitals is approximately a factor of two smaller, consistent with the type of argument presented above which would imply proportionality to $\left\langle n\left|L_{z}^{2}\right| n\right\rangle$ in that case.

We expect the dependence of quasiparticle energies on the magnitude and orientation of the order parameter, and also on external fields discussed below, to change in character when the typical matrix element becomes comparable to the level spacing, i.e. when

$$
\frac{0.14 \xi_{d}^{2}}{\delta \mathcal{N}_{a}}=\delta
$$




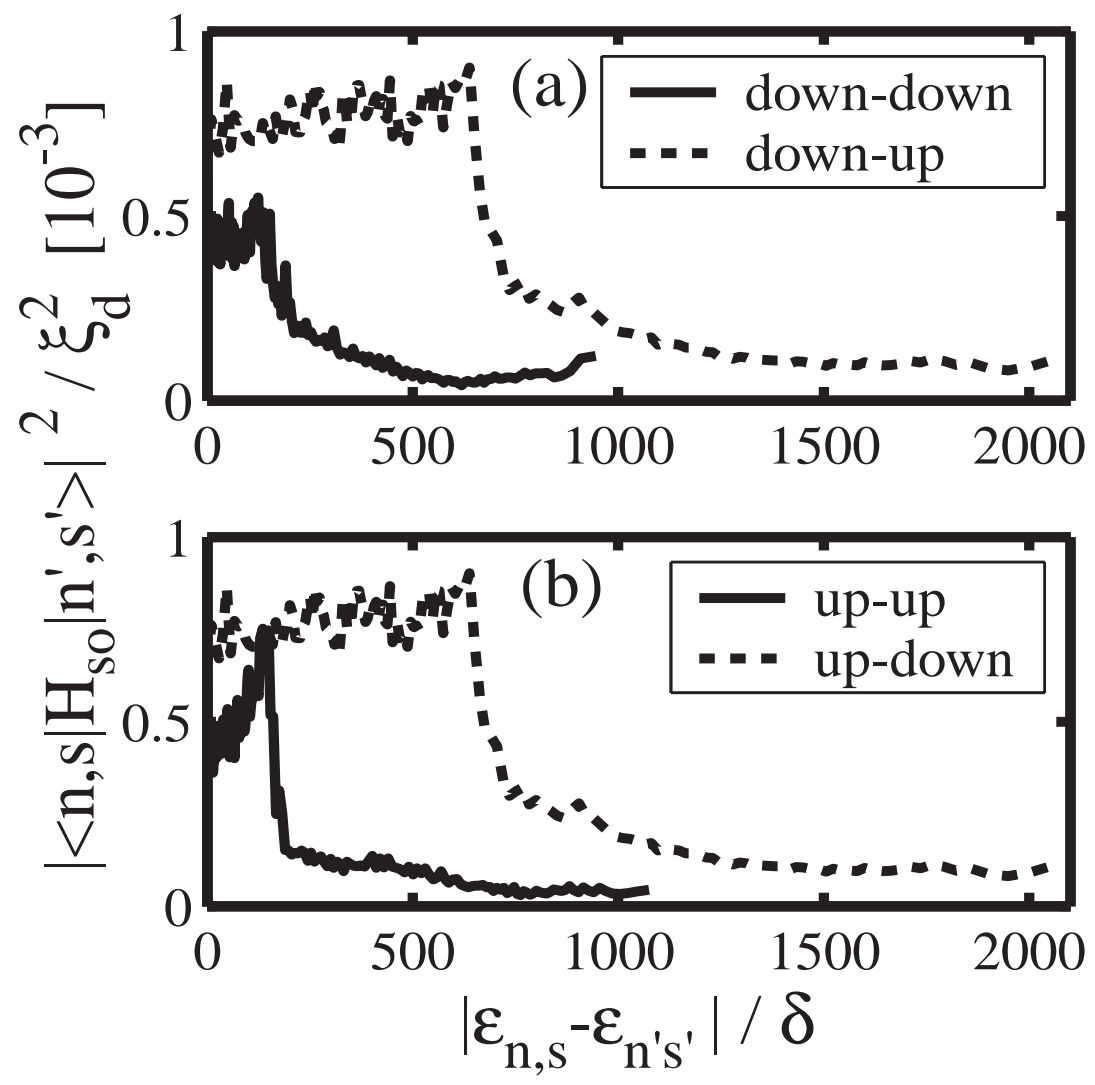

FIG. 7: Average squared matrix elements of the spin-orbit interaction vs. energy difference for a polarized 143-atom nanoparticle. $|n, s\rangle$ and $\left|n^{\prime}, s^{\prime}\right\rangle$ are quasi-particle states without spin-orbit interactions. The four curves correspond to the four possible spin combinations.

Our numerical calculations of quasiparticle spectra are consistent with using the condition $\hbar \tau_{S O}^{-1} \sim \epsilon_{S O}=\delta$ as a criterion for the start of the crossover to the strong coupling limit, and the condition that the typical avoided crossing gap estimate be equal to $\delta$, as a criterion for completion of the crossover to strong-coupling relatively rigid spectra.

For Cobalt nanoparticles the later condition is reached for $\mathcal{N}_{a} \sim 2000$; for smaller nanoparticles, the quasiparticles generally have somewhat well-defined spin character, some Poisson character in their spectral statistics, and complicated evolution patterns with external field and order parameter variations. For larger nanoparticles, which we are not however able to study numerically, we expect that quasiparticles will have strongly mixed spins, and more rigid spectra with smoother evolution patterns. All the ferromagnetic nanoparticles that we are able to study here, and many nanoparticles studied experimentally, are in the crossover regime. Note that since $\delta \propto W_{d} / \mathcal{N}_{a},\left(\xi_{d}^{2} /\left(\delta \mathcal{N}_{a}\right) \propto \hbar \tau_{S O}^{-1}\right.$; the two conditions differ 


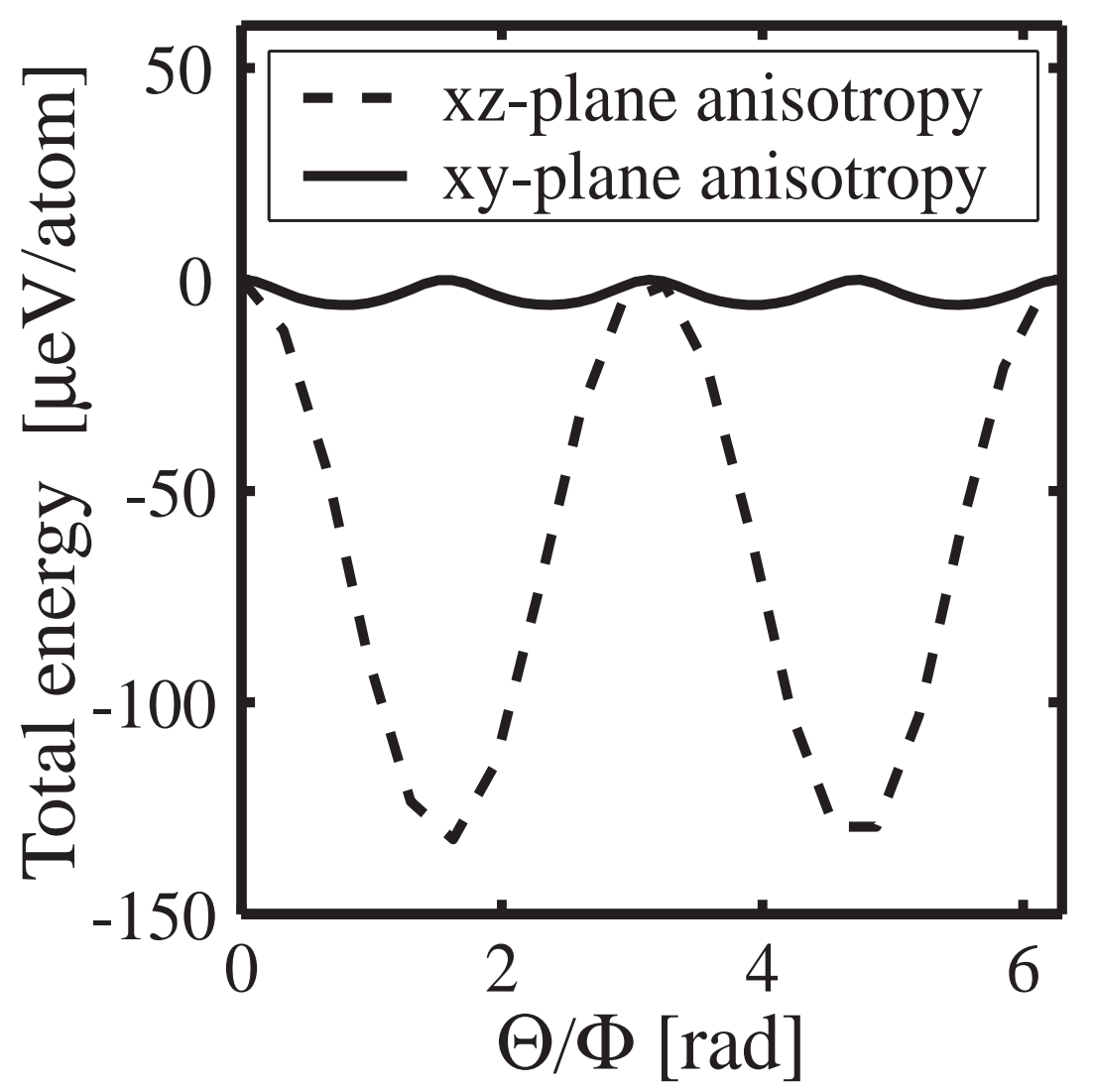

FIG. 8: Magnetic anisotropy energies for a 143-atom hemispherical nanoparticle. The dotted line represents $\left[E\left(h_{\hat{z}}^{\star} \hat{z}\right)-E\left(h_{\hat{\Omega}}^{\star} \hat{\Omega}\right)\right]$ vs. the angle $\Theta$ between $\hat{\Omega}$ and $\hat{z}$, when $\hat{\Omega}$ is in the $z x$-plane. The black line represents $\left[E\left(h_{\hat{x}}^{\star} \hat{x}\right)-E\left(h_{\hat{\Omega}}^{\star} \hat{\Omega}\right)\right]$ vs. the angle $\Phi$ between $\hat{\Omega}$ and $\hat{x}$, when $\hat{\Omega}$ lies in the $x y$-plane.

quantitatively not parametrically.

The total ground-state energy of the particle $E\left(h_{\hat{\Omega}}^{\star} \hat{\Omega}\right)$, obtained by summing over the lowest $N$ occupied orbitals, depends on the direction of the self-consistent spin-splitting field $\hat{\Omega}$. In Fig. [8] we plot $E\left(h_{\hat{z}}^{\star} \hat{z}\right)-E\left(h_{\hat{\Omega}}^{\star} \hat{\Omega}\right)$ vs. the angle $\Theta$ between $\hat{\Omega}$ and $\hat{z}$ (when $\hat{\Omega}$ lies in the $z x$-plane) and $E\left(h_{\hat{x}}^{\star} \hat{x}\right)-E\left(h_{\hat{\Omega}}^{\star} \hat{\Omega}\right)$ vs. the angle $\Phi$ between $\hat{\Omega}$ and $\hat{x}$ (when $\hat{\Omega}$ lies in the $z x$-plane). From the figure we can see that the $x y$-plane is almost an easy plane for the model nanoparticle, except for a weak energy dependence which generates four easy axis in the directions $( \pm \hat{x} \pm \hat{y}) / 2$. Again this property reflects the large significance of the overall sample shape. The four easy axis directions are remnants of the magnetic anisotropy symmetry in bulk f.c.c. ferromagnets. Group theory considerations demand that a bulk f.c.c. ferromagnet have an easy axis in one of the directions perpendicular to the 
$8( \pm 1, \pm 1, \pm 1)[6]$ planes. In a hemispherical nanoparticle the f.c.c. symmetry is partially lifted and the magnetization is forced to lie in the $x y$-plane. We can define the following two anisotropy-energy-per-atom constants

$$
\begin{aligned}
k(\hat{z}, \hat{x}) \equiv \frac{E\left(h_{\hat{z}}^{\star} \hat{z}\right)-E\left(h_{\hat{x}}^{\star} \hat{x}\right)}{\mathcal{N}_{a}} \approx 0.13 \mathrm{meV}, \\
k(\hat{x}, \hat{y}) \equiv \frac{E\left(h_{\hat{x}}^{\star} \hat{x}\right)-E\left(h_{\hat{y}}^{\star} \hat{y}\right)}{\mathcal{N}_{a}} \approx 0.01 \mathrm{meV} .
\end{aligned}
$$

As expected on the basis of the qualitative considerations of Section [IIA, the anisotropy per occupied orbital is much smaller than the average single-particle level shift $\epsilon_{\mathrm{SO}}$ (approximately 200 times smaller in both cases) due to cancellations between positive and negative shifts mentioned above.

We notice that $k(\hat{z}, \hat{x})$ for our nanoparticle is larger than the bulk anisotropy per atom $k_{\text {bulk }}=60 \mu \mathrm{eV}$, as one would expect because of the hemispherical shape of the sample. However $k(\hat{x}, \hat{y})$ is actually smaller than $k_{\text {bulk }}$. This comparison should be regarded with caution, since it is known that accurate theoretical estimates of the magnetic anisotropy for bulk crystals are very delicate and agreement with experiment values even in the bulk is still not completely satisfactory [35];

we have not evaluated the anisotropy energy that results from the bulk limit of our nanoparticle model and it may well not agree with experiment. Nonetheless, the small value that we find for the anisotropy in the $x y$-plane might be connected to the puzzling finding, in both tunneling[7] and switching-field[3] experiments on single ferromagnetic nanoparticles, of anisotropy energies per atom a factor of five smaller than bulk values.

\section{FLUCTUATIONS OF MAGNETIC ANISOTROPY}

So far we have considered only average values of the anisotropy constants. In ferromagnetic nanoparticles, however, these quantities are also characterized by large fluctuations as

a function of experimentally relevant parameters. Anisotropy fluctuations are the topic of this section. 


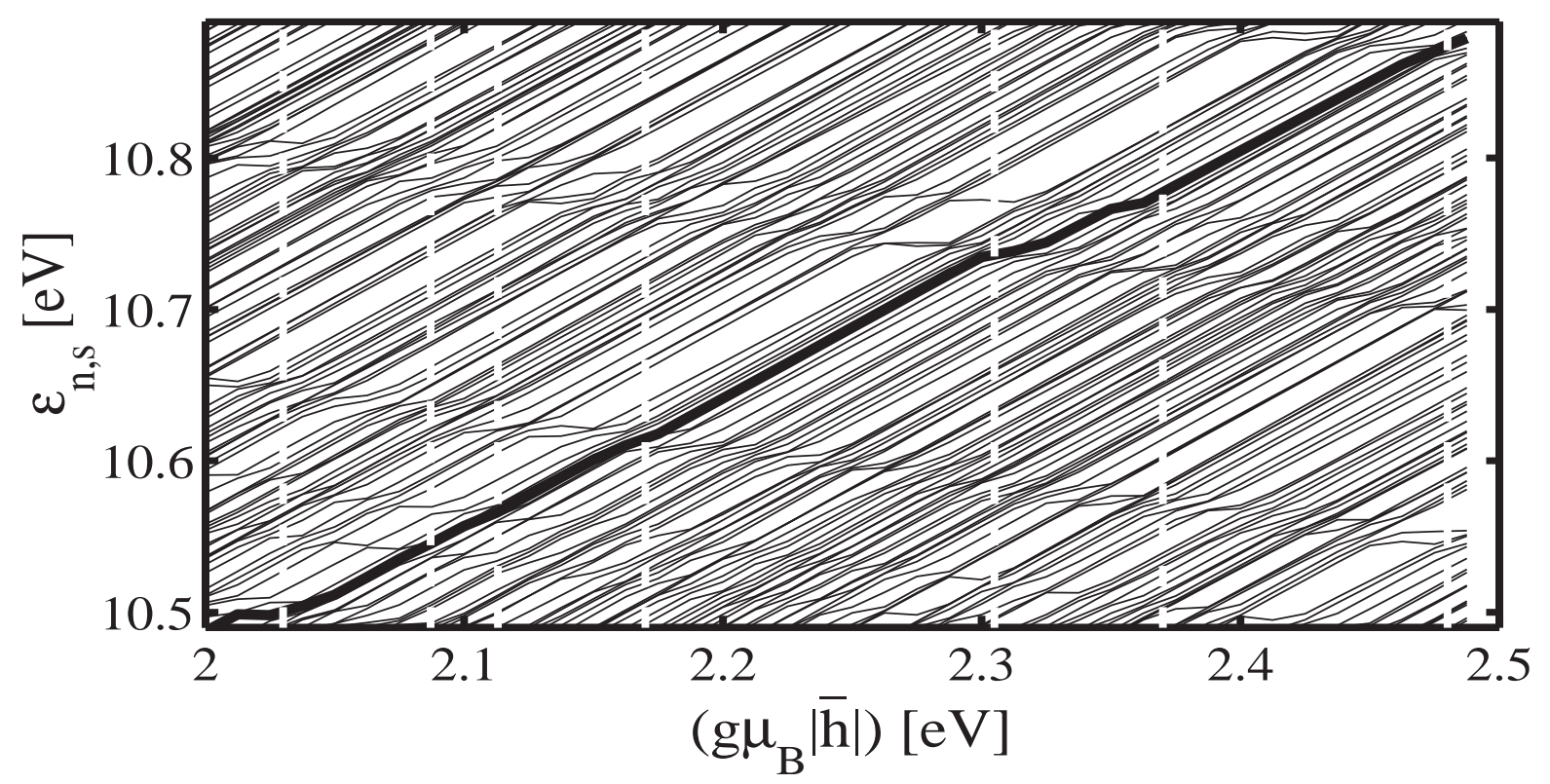

FIG. 9: Change of quasiparticle energies with increasing spin-splitting field $|\vec{h}|$ near $h^{\star}$ for a 143atom nanoparticle. 200 levels are shown. The Fermi level is the thick black line. The vertical white dashed lines indicate the positions of the charge redistributions displayed in Fig. [10]. The vertical black dashed line corresponds to the self-consistent spin-splitting field.

\section{A. Charge-induced fluctuations as a function of the spin-splitting field}

When spin-orbit interactions are included, all quasi-particle eigenstates have mixed majority-spin and minority-spin character.

Our specific calculations, however, are for small enough particles such that most eigenstates still have predominantly one spin character. It is convenient to use this "predominant spin" to label the states when discussing their dependence on $|\vec{h}|$ or on an external magnetic field. In a paramagnetic system $\left(\vec{h}^{\star}=0\right)$ in absence of external field, there is a Kramer degeneracy that pairs up eigenstates with opposite predominant spin character. The degeneracy is lifted in the ferromagnetic state. Majority-spin states will move down in energy while minority-spin states will move up as $|\vec{h}|$ increases. Because of spin-orbit-induced level repulsion, all of the level crossings are avoided; the levels evolve continuously with $|\vec{h}|$, gradually changing their spin and orbital character. In Fig. [9] we plot the variation of two hundred levels as a function of $g_{s} \mu_{\mathrm{B}}|\vec{h}|$ near $g_{s} \mu_{\mathrm{B}} h^{\star}=2.2 \mathrm{eV}$, for a 143-atom nanoparticle. The Fermi level is the thick black line lying in a region of predominantly minority-spin quasiparticles 


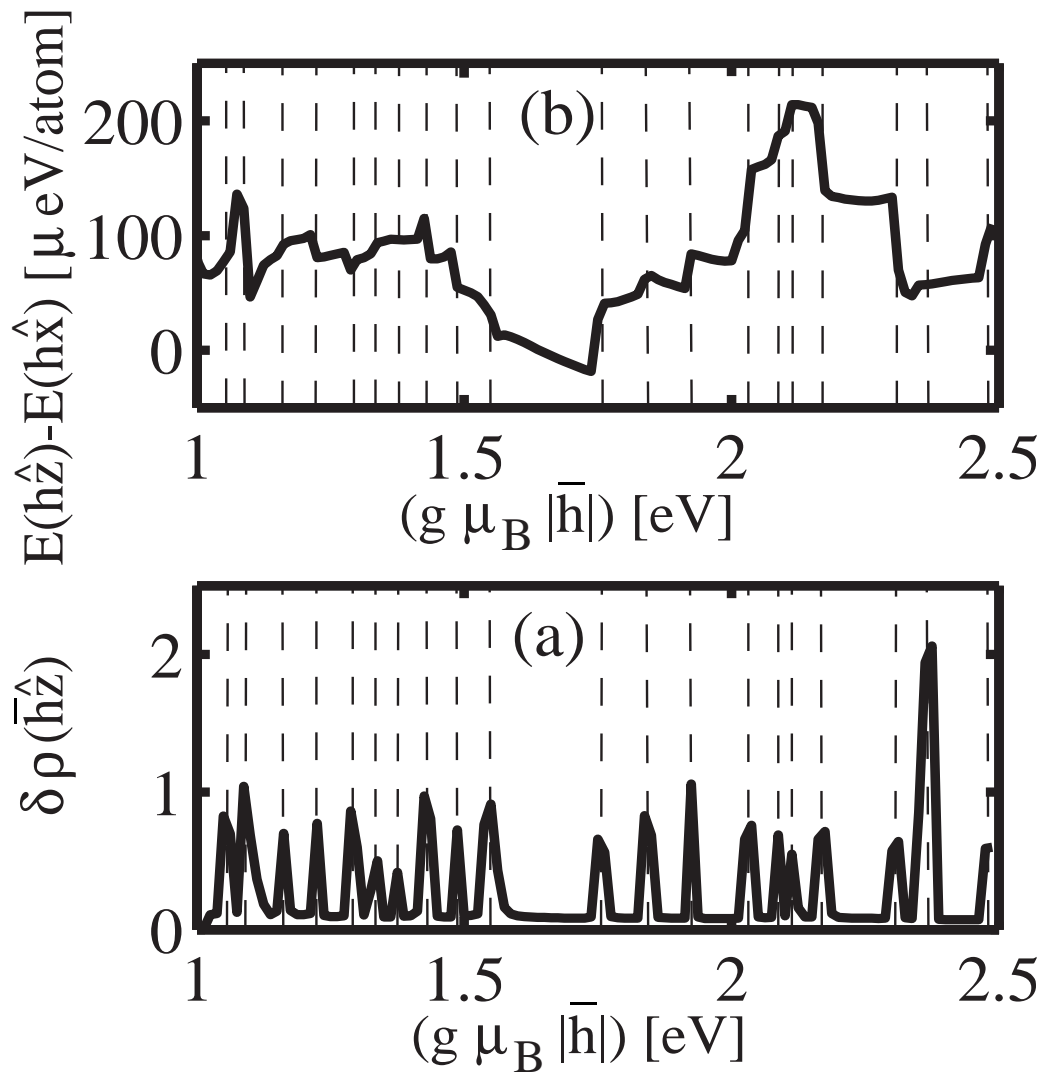

FIG. 10: Relation between charge fluctuations and anisotropy in 143-atom nanoparticle. (a) Total charge fluctuation defined in Eq. [19]. Charge fluctuations of the order of one electron result in fluctuations in the magnetic anisotropy. (b) Anisotropy energy in the $z x$-plane vs. the magnitude of the spin-splitting field taken as a free parameter.

(lines with positive slope). By increasing $|\vec{h}|$, individual majority-spin quasiparticle energies (negative slopes) come down from regions above the Fermi level, creating avoided crossing gaps as they approach minority-spin quasiparticles moving in the opposite direction. Since a 143-atom nanoparticle is still toward the weak spin-orbit coupling limit, the level crossings will be avoided only weakly. Whenever one of the majority-spin quasiparticles crosses the Fermi level, there is a change in the spin character of one of the quasiparticle levels and the total spin of the nanoparticle increases approximately by one. Due to the exchange interaction, such spin-flips bring about charge redistribution inside the nanoparticle, which in turns gives rise to fluctuations in the anisotropy energy. In Fig. [10a] we plot the total 
atomic site charge redistribution, when $h \equiv|\vec{h}| \Rightarrow h+\Delta h$, for $\Delta h=12.5 \mathrm{meV}$

$$
\delta \rho(h)=\sum_{i}\left|\rho_{i}(h+\Delta h)-\rho_{i}(h)\right| \approx \sum_{i}\left|\frac{d \rho_{i}(h)}{d h}\right| \Delta h,
$$

where $\rho_{i}(h)$ is the total charge at atom $i$. It is seen that $\delta \rho(h)$ changes by one in the small interval $\Delta h=12.5 \mathrm{meV}$ where majority-spin quasiparticles weakly avoid crossing the Fermi level. (Note that this is a charge redistribution, not a change in total charge.) This result is not unexpected, since the majority and minority spin orbitals should have uncorrelated spatial distributions. The charge redistribution is likely overstated by our simplified model, however, since it does not account for long-range Coulomb interactions and related screening effects. It is, however still a relatively small $\sim 1 / N$ effect. The anisotropy energy fluctuations associated with the level crossings that occur as a function of $h$, shown in Fig. [10]b] are much larger in relative terms.

Variation in $h$ corresponds to variation in the amplitude of the magnetic order parameter. In micromagnetic modeling of nanoparticles, it is implicitly assumed that the magnitude of the order parameter is fixed and that this collective degree of freedom can be ignored in modeling nanoparticle properties. The substantial dependence of anisotropy energy on $h$ that we find demonstrates that the amplitude and orientation fluctuations of the order parameter can be strongly coupled in small magnetic nanoparticles. Related anisotropy fluctuations as a function of electron number are important in understanding the additionpotential-spectroscopy single-electron-transistor experiments[7] which are the topic of the next section.

\section{B. Mesoscopic fluctuations as a function of electron number}

The analysis of the field-dependence of tunneling resonances in experiments on magnetic nanoparticles suggests that the anisotropy energy fluctuates significantly from eigenstate to eigenstate [7, 9]. In Refs. [7, 9] the effects of these fluctuations were mimicked by using two different anisotropy energy constants $k_{N}$ and $k_{N \pm 1}=k_{N}+\delta k_{ \pm}$for $N$ - and $N \pm 1$-electron states. By assuming $\delta k_{ \pm} / k_{N}$ in the range of a few percent, it was possible to explain the non-monotonic behavior of the tunneling resonances seen experimentally.

Within our microscopic model, we have calculated $k_{N}$ and $k_{N \pm 1}$ as defined in Eqs. (17, 18). In light of results of the type summarized in Fig. [8], it suffices to compute the total energy 
for two different directions of the spin-splitting field and take the difference. The total energy for a $N$-electron system, for spin splitting fields along directions $\hat{\Omega}_{i}, i=1,2$, is given by:

$$
E\left(h_{i}^{\star} \hat{\Omega}_{i}\right)=\sum_{n=1}^{N} \epsilon_{n}\left(h_{i}^{\star} \hat{\Omega}_{i}\right)+\frac{\left(h_{i}^{\star}\right)^{2}}{2 U_{d d}} \mathcal{N}_{a}, \quad i=1,2,
$$

where $h_{i}^{\star}$ is calculated self-consistently for a given fixed direction. The anisotropy-energyper-atom constant is then

$$
k_{N}\left(\hat{\Omega}_{1}, \hat{\Omega}_{2}\right)=\frac{1}{\mathcal{N}_{a}}\left[\sum_{n=1}^{N} \epsilon_{n}\left(h_{1}^{\star} \hat{\Omega}_{1}\right)-\epsilon_{n}\left(h_{2}^{\star} \hat{\Omega}_{2}\right)\right]+\frac{\left(h_{1}^{\star}\right)^{2}-\left(h_{2}^{\star}\right)^{2}}{2 U_{d d}}\left(g_{s} \mu_{\mathrm{B}}\right)^{2}
$$

where the subscript $N$ emphasizes the fact that the constant refers to a $N$-electron system. It turns out that the value of $h^{\star}$ depends very weakly on $\hat{\Omega}$. For example, in a 143-atom nanoparticle $\left|h_{1}^{\star}-h_{2}^{\star}\right| / h_{1}^{\star} \approx 10^{-3}$. This property reflects the large ratio between the total magnetic condensation energy and the anisotropy energy, even for nanoparticles. The strong coupling between amplitude and orientation fluctuations mentioned above, occurs only when a small change in $h$ leads to a crossing between majority and minority spin orbitals. Evaluating $E\left(h \hat{\Omega}_{2}\right)$ at $h=h_{1}^{\star}=h_{2}^{\star}+\delta h^{\star}$, expanding in powers of $\delta h^{\star} / h_{1}^{\star}$ and remembering that $h_{2}^{\star}$ minimizes $E\left(h \hat{\Omega}_{2}\right)$ yields

$$
k_{N}\left(\hat{\Omega}_{1}, \hat{\Omega}_{2}\right)=\frac{1}{\mathcal{N}_{a}}\left[\sum_{n=1}^{N} \epsilon_{n}\left(h_{1}^{\star} \hat{\Omega}_{1}\right)-\epsilon_{n}\left(h_{1}^{\star} \hat{\Omega}_{2}\right)\right]+\mathcal{O}\left[\frac{N}{\mathcal{N}_{a}}\left(\frac{\delta h^{\star}}{h_{1}^{\star}}\right)^{2}\right]
$$

for any $N$.

Adding an electron to the system changes the magnitude of the spin-splitting field $h_{i}^{\star} \rightarrow$ $h_{i}^{\star}$, but again $\left|h_{i}^{\star}-h_{i}^{\star}\right| / h_{i}^{\star}<<1$. Thus we obtain

$$
\begin{array}{r}
k_{N+1}\left(\hat{\Omega}_{1}, \hat{\Omega}_{2}\right)=\frac{1}{\mathcal{N}_{a}}\left[\sum_{n=1}^{N+1} \epsilon_{n}\left(h_{1}^{\star} \hat{\Omega}_{1}\right)-\epsilon_{n}\left(h_{1}^{\star} \hat{\Omega}_{2}\right)\right]+\mathcal{O}\left[\frac{N+1}{\mathcal{N}_{a}}\left(\frac{\delta h^{\star}}{h_{1}^{\star}}\right)^{2}\right] \\
=k_{N}\left(\hat{\Omega}_{1}, \hat{\Omega}_{2}\right)+\frac{\epsilon_{N+1}\left(h_{1}^{\star} \hat{\Omega}_{1}\right)-\epsilon_{N+1}\left(h_{1}^{\star} \hat{\Omega}_{2}\right)}{\mathcal{N}_{a}}+\mathcal{O}\left[\frac{N+1}{\mathcal{N}_{a}}\left(\frac{\delta h^{\star}}{h_{1}^{\star}}\right)^{2}\right]
\end{array}
$$

Replacing

$$
\frac{\epsilon_{N+1}\left(h_{1}^{\star} \hat{\Omega}_{1}\right)-\epsilon_{N+1}\left(h_{1}^{\star} \hat{\Omega}_{2}\right)}{\mathcal{N}_{a}} \approx \frac{\Delta\left[\delta \epsilon_{N+1}\left(\hat{\Omega}_{1}, \hat{\Omega}_{2}\right)\right]}{\mathcal{N}_{a}}
$$

where $\Delta\left[\delta \epsilon_{N+1}\left(\hat{\Omega}_{1}, \hat{\Omega}_{2}\right)\right]$ is the width of the distribution of the single-particle anisotropies in plane containing the two directions $\hat{\Omega}_{1}$ and $\hat{\Omega}_{2}$, we finally obtain

$$
k_{N+1}\left(\hat{\Omega}_{1}, \hat{\Omega}_{2}\right) \approx k_{N}\left(\hat{\Omega}_{1}, \hat{\Omega}_{2}\right)+\frac{\Delta\left[\delta \epsilon_{N+1}\left(\hat{\Omega}_{1}, \hat{\Omega}_{2}\right)\right]}{\mathcal{N}_{a}}
$$



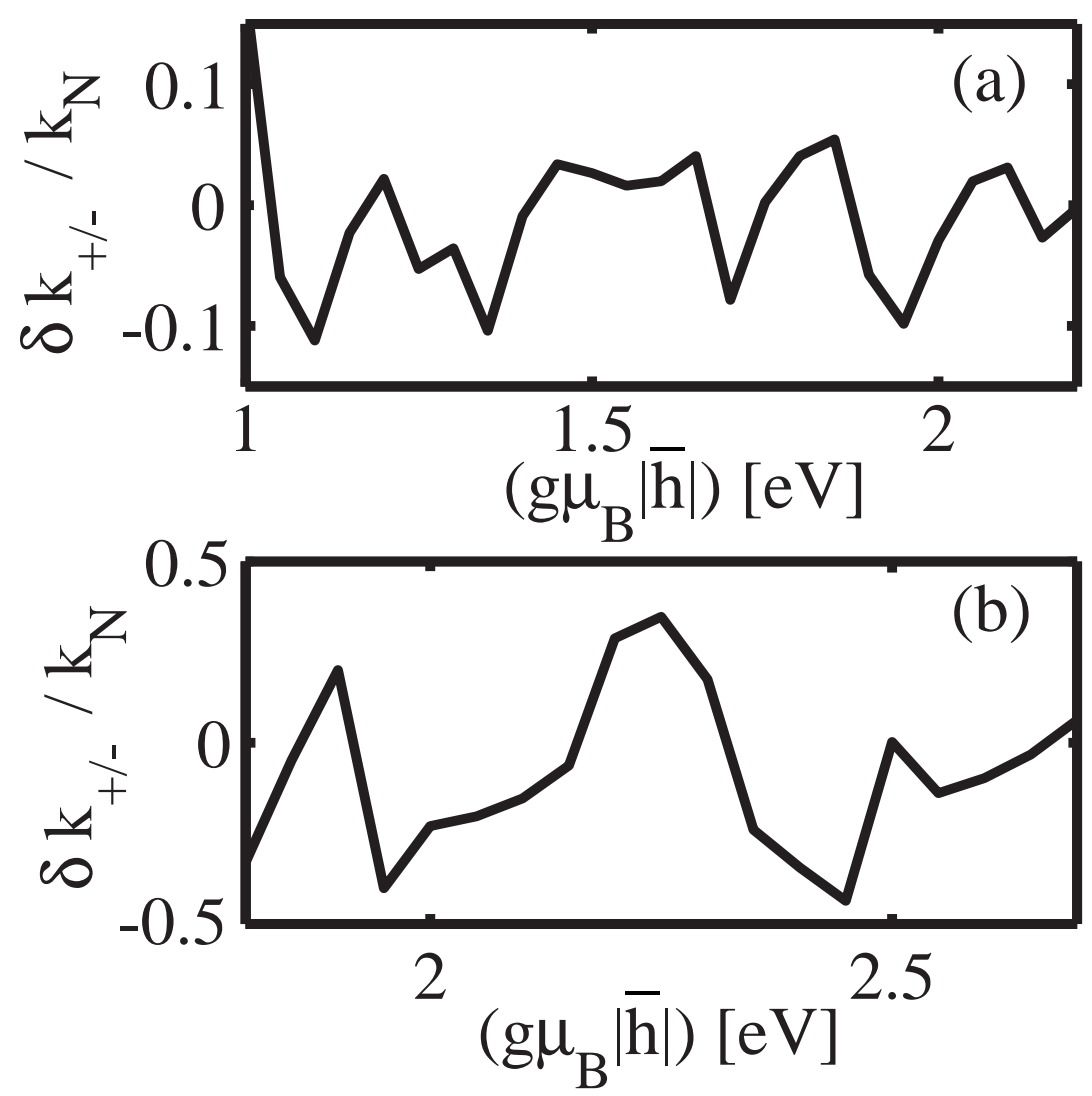

FIG. 11: Relative shift $\delta k_{ \pm} / k_{N} \equiv\left(k_{N+1}-k_{N}\right) / k_{N}$ of the anisotropy energy constant $k_{N}(\hat{z}, \hat{x})$, when the electron number increases by one, $N \rightarrow N+1$, as a function of spin-splitting field. (a) 260-atom nanoparticle. (b) 143-atom nanoparticle. The self-consistent spin-splitting field is $h^{\star} \approx 2.2 \mathrm{eV}$ for both a 143 -atom and a 260 -atom nanoparticle.

The fluctuations of $k_{N}$ due to an additional electron are therefore of the order of $\Delta\left[\delta \epsilon_{N+1}\left(\hat{\Omega}_{1}, \hat{\Omega}_{2}\right)\right] / \mathcal{N}_{a}$, and are regulated by the mean level spacing, $\delta$, which suppresses the magnitude of $\Delta\left[\delta \epsilon_{N+1}\left(\hat{\Omega}_{1}, \hat{\Omega}_{2}\right)\right]$ at large nanoparticle sizes as we discuss below.

For a 143 -atom dot, $\Delta\left[\delta \epsilon_{N+1}\left(\hat{\Omega}_{1}, \hat{\Omega}_{2}\right)\right] \approx 2.9 \mathrm{meV}$ in the zx-plane, where $k_{N}(\hat{z}, \hat{x}) \approx 0.13$ meV. Therefore $\delta k_{ \pm} / k_{N}(\hat{z}, \hat{x})=\frac{1}{\mathcal{N}_{a}} \Delta\left[\delta \epsilon_{N+1}\left(\hat{\Omega}_{1}, \hat{\Omega}_{2}\right)\right] / k_{N}(\hat{z}, \hat{x}) \approx 15 \%$. These estimates are confirmed by a direct calculation of $k_{N}(\hat{z}, \hat{x})$ and $k_{N+1}(\hat{z}, \hat{x})$ for 143-atom and 260-atom nanoparticles, shown in Fig. [11]. Here $k_{N}(\hat{z}, \hat{x})$ and $k_{N+1}(\hat{z}, \hat{x})$ are plotted as a function of $g_{s} \mu_{\mathrm{B}} h$, taken as a free parameter (the self-consistent value is close to $g_{s} \mu_{\mathrm{B}} h^{\star}=2.2 \mathrm{eV}$ for both a 143-atom and a 260-atom nanoparticle). For a 143-atom nanoparticle the calculated anisotropy energy fluctuations are of the order of 20\%. For a 260-atom nanoparticle the anisotropy energy fluctuations are smaller, but still close to $5 \%$. 
We can understand the surprisingly large fluctuations in anisotropy energy with particle and electron number by the following observations. Our numerical results (see Fig. 3 and Fig. 5) indicate that the contribution of a given orbital to the anisotropy energy is chosen essentially at random from a distribution which, for the $z x$-plane anisotropy, has a width $\Delta\left(\delta \epsilon_{n, s}\right) \sim 2.2 \xi_{d}^{2} / W_{d} \sim 2.9 \mathrm{meV}$ and a much smaller mean value $\left\langle\delta \epsilon_{n, s}\right\rangle=$ $\left\langle\epsilon_{n, s}\left(h^{\star} \hat{z}\right)-\epsilon_{n, s}\left(h^{\star} \hat{x}\right)\right\rangle=\mathcal{N}_{a} k_{N}(\hat{z}, \hat{x}) / N \sim 15 \mu \mathrm{eV}$, where $k_{N}(\hat{z}, \hat{x}) \sim 0.13 \mathrm{meV}$. The relative change in the anisotropy energy expected when one additional orbital is occupied is $\sim 4.3 \xi_{d}^{2} / W_{d} /\left(k_{N}(\hat{z}, \hat{x}) \mathcal{N}_{a}\right)$, which can easily be larger than $1 \%$ for $\mathcal{N}_{a} \leq 1500$.

We expect strong level repulsion in the rigid spectrum of larger nanoparticles to be accompanied by more regular behavior of the anisotropy energy per atom, with smaller variations as a function $\vec{h}^{\star}$, and electron number.

We note that fluctuations in the contribution of a given orbital to the anisotropy energy should not exceed $\sim \delta$, which vanishes in the limit of very large particles. We expect that the distribution function of contributions to anisotropy from orbitals near the Fermi energy to become narrow when $\delta$ is smaller than the average anisotropy energy contribution $\sum 10 \mu \mathrm{eV}$. This condition is satisfied for particles containing more than $\sim 10^{5}$ atoms.

\section{Anisotropy energy dependence on particle atom number and shape}

We conclude this section with a few remarks on the dependence of the magnetic anisotropy energy on nanoparticle size and shape. In Fig. [12] we plot the anisotropy energy per atom for nanoparticles of different sizes. For a small number of atoms (below 60) the anisotropy per atom is very large and decreases rapidly with size. In this regime, nonextensive surface contributions, which are present because of the abrupt truncation of the lattice, are clearly playing a dominating role. By increasing the size of the nanoparticle, we must eventually reach a regime where total magnetic anisotropy becomes proportional to the nanoparticle volume. Fig. [12] shows that this regime is not yet fully established even for nanoparticles with hundreds of atoms, and fluctuations are still pronounced. In this regime the anisotropy per atom is still 2-3 times larger than the bulk value for Cobalt, which is $0.06 \mathrm{meV}$. There is no regime in which bulk (proportional to $\mathcal{N}_{a}$ ) and surface (proportional to $\mathcal{N}_{a}^{2 / 3}$ ) contributions to the anisotropy can be cleanly separated.

In is clear that for small particles the shape of the nanoparticle plays an important role 


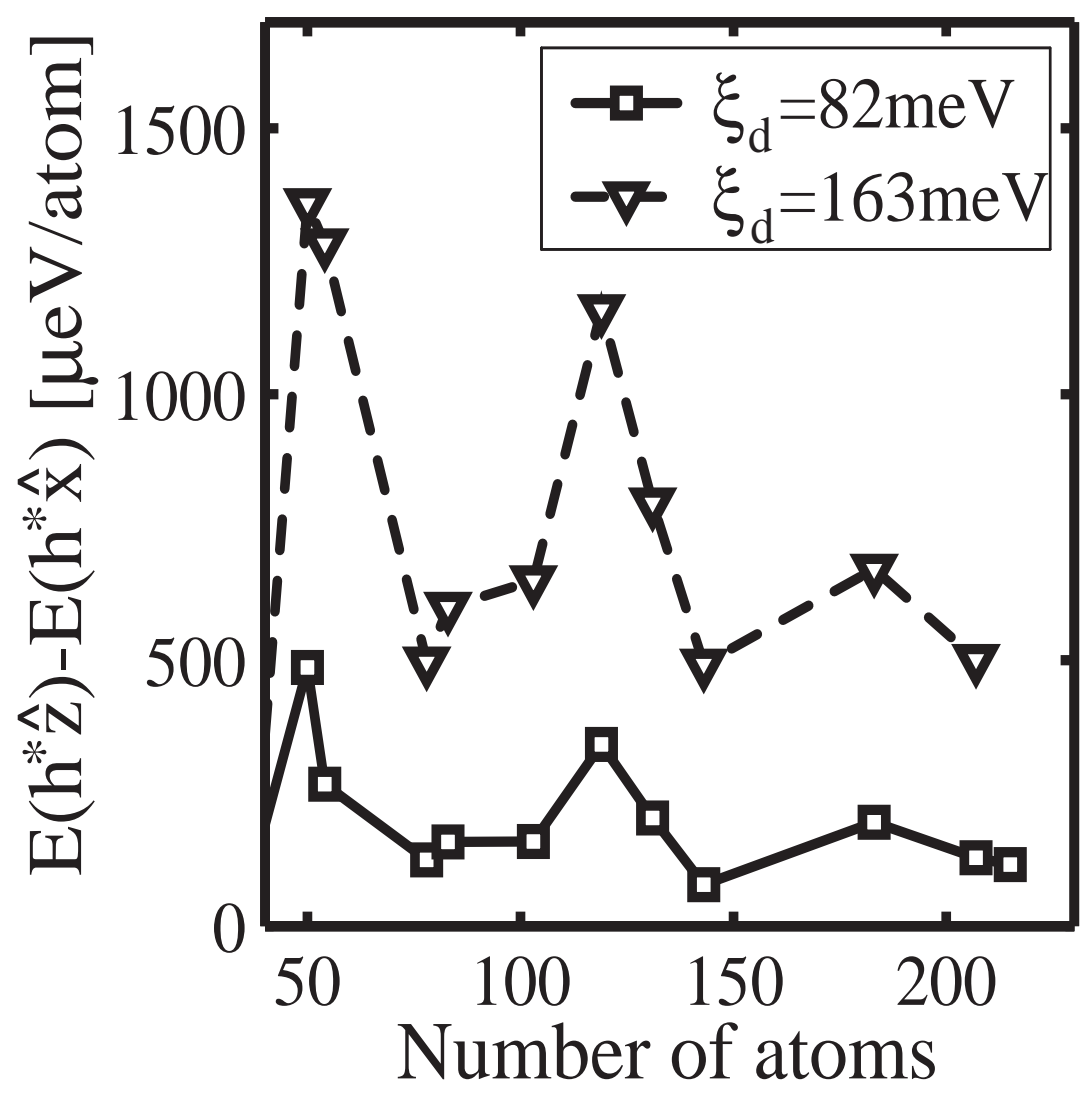

FIG. 12: Anisotropy energy as a function of nanoparticle size for two values of the spin-orbit coupling $\xi_{d}$. A splitting field of $2 \mathrm{eV}$ is assumed for all sizes.

in the determination of the magnetic anisotropy. For example, as seen in Fig. [13], for spherical particles we find anisotropy energies which are one order of magnitude smaller than the $z x$ anisotropy of hemispherical particles, comparable instead to the $x y$ anisotropy of hemispherical particles with the same number of atoms. A 140-atom spherical particle has an anisotropy energy per atom $\approx 0.01-0.02 \mathrm{meV}$.

It is interesting to notice that both tunneling[7] and switching-field[3] experiments on single ferromagnetic nanoparticles find anisotropy energies per atom which are of the order $0.01 \mathrm{meV}$, a factor of five smaller than the bulk value. The nanoparticles in tunneling experiments are roughly hemispherical [1, 7], whereas the nanoparticle shape in Ref. [3] is close to spherical.

Further theoretical studies that focus on the relationship between nanoparticle shape and anisotropy could be helpful to efforts to engineer ferromagnetic nanoparticles whose shape, size, and crystal structure are tuned to produce desired magnetic properties. 


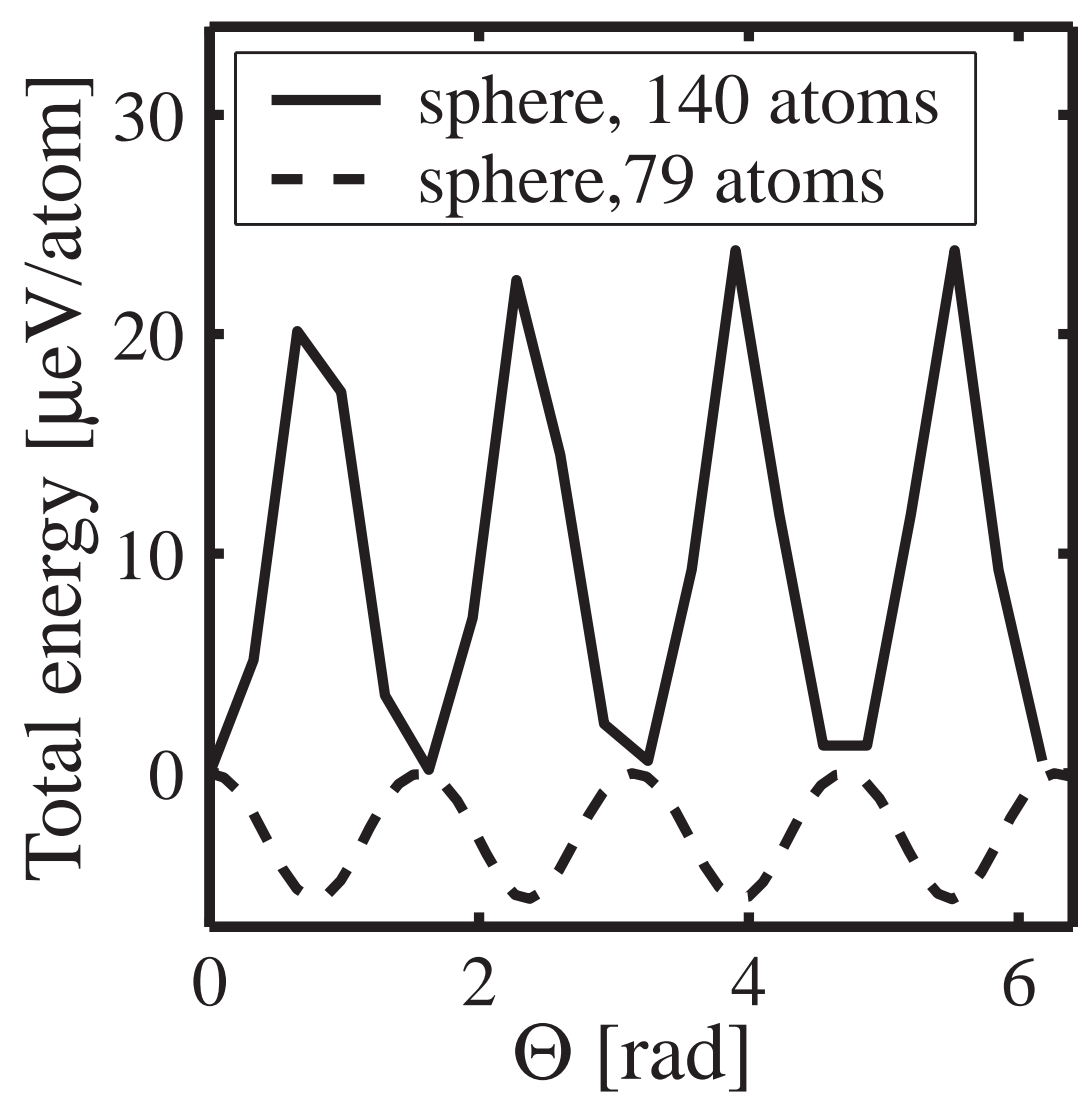

FIG. 13: Magnetic anisotropy energy in the $z x$-plane, $\left[E\left(h_{\hat{z}}^{\star} \hat{z}\right)-E\left(h_{\hat{\Omega}}^{\star} \hat{\Omega}\right)\right]$ for spherical nanoparticles of two sizes. $\Theta$ is the angle between $\hat{z}$ and $\hat{\Omega}$. By symmetry, the anisotropy energy in the $x y$-plane is now the same as in the $z x$-plane. The spin-splitting field is assumed to be $2 \mathrm{eV}$ for both sizes.

V. HYSTERESIS AND VARIATION OF SINGLE-PARTICLE LEVELS IN AN EXTERNAL MAGNETIC FIELD

In the last section we shall investigate the effect of an external magnetic field on the total energy of the nanoparticle. This will allow us to make a connection between our microscopic model and more familiar classical micromagnetic energy functional expressions which are normally used to interpret the results of switching-field experiments[3]. We shall also study the dependence of the quasiparticle energy levels on external magnetic fields; this complex behavior has been probed directly in recent tunneling experiments [1, []. 


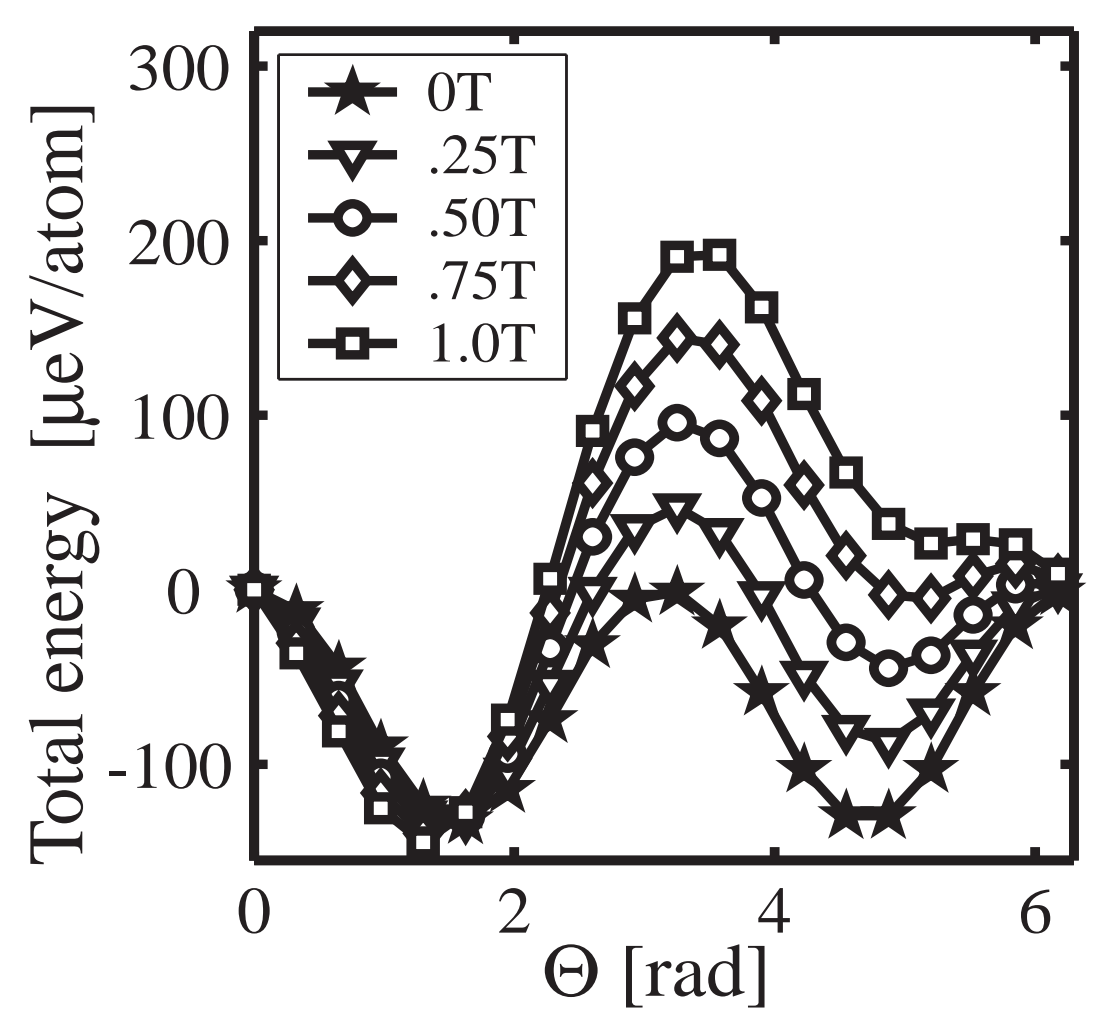

FIG. 14: "Uniaxial" anisotropy energy as a function of the direction of the spin-splitting field $\hat{\Omega}$, for different magnitudes of the external magnetic field. The external field is in the $z x$-plane at an angle $\theta=\pi / 4$ with the $\hat{z}$-axis. $\Theta$ is angle between $\hat{\Omega}$ (lying in the $z x$-plane) and $\hat{z}$.

\section{A. Hysteresis}

Ideally one would like to study the three dimensional energy landscape $E\left(\vec{h}^{\star}, \vec{H}_{\text {ext }}\right)$ as a function of $\hat{\Omega}(\Theta, \Phi) \equiv \vec{h}^{\star} /\left|\vec{h}^{\star}\right|$, for several values of $\vec{H}_{\text {ext }}$. Here we consider two simplified cases. In Fig. [14] we plot the total energy difference $E\left(\vec{h}_{\hat{z}}^{\star} \hat{z}\right)-E\left(\vec{h}_{\hat{\Omega}}^{\star} \hat{\Omega}\right)$ as a function of an external magnetic field $\vec{H}_{\text {ext }}$. Here $\hat{\Omega}$ is allowed to rotate in the $z x$-plane. In this picture we recognize the familiar features of a micromagnetic energy functional characterized by a uniaxial magnetic anisotropy: at $\vec{H}_{\text {ext }}=0$ there are two degenerate minima, separated by an energy barrier, representing two equivalent easy directions $\pm \hat{x}$. When a magnetic field is applied in the $z x$-plane, the degeneracy is lifted and the minimum in the $-\hat{x}$ direction becomes a metastable local minimum, until a switching field is reached at which the local minimum disappears. This happens at $\left|\vec{H}_{\text {ext }}\right| \approx 1 \mathrm{~T}$ for a 143 -atom nanoparticle. This 


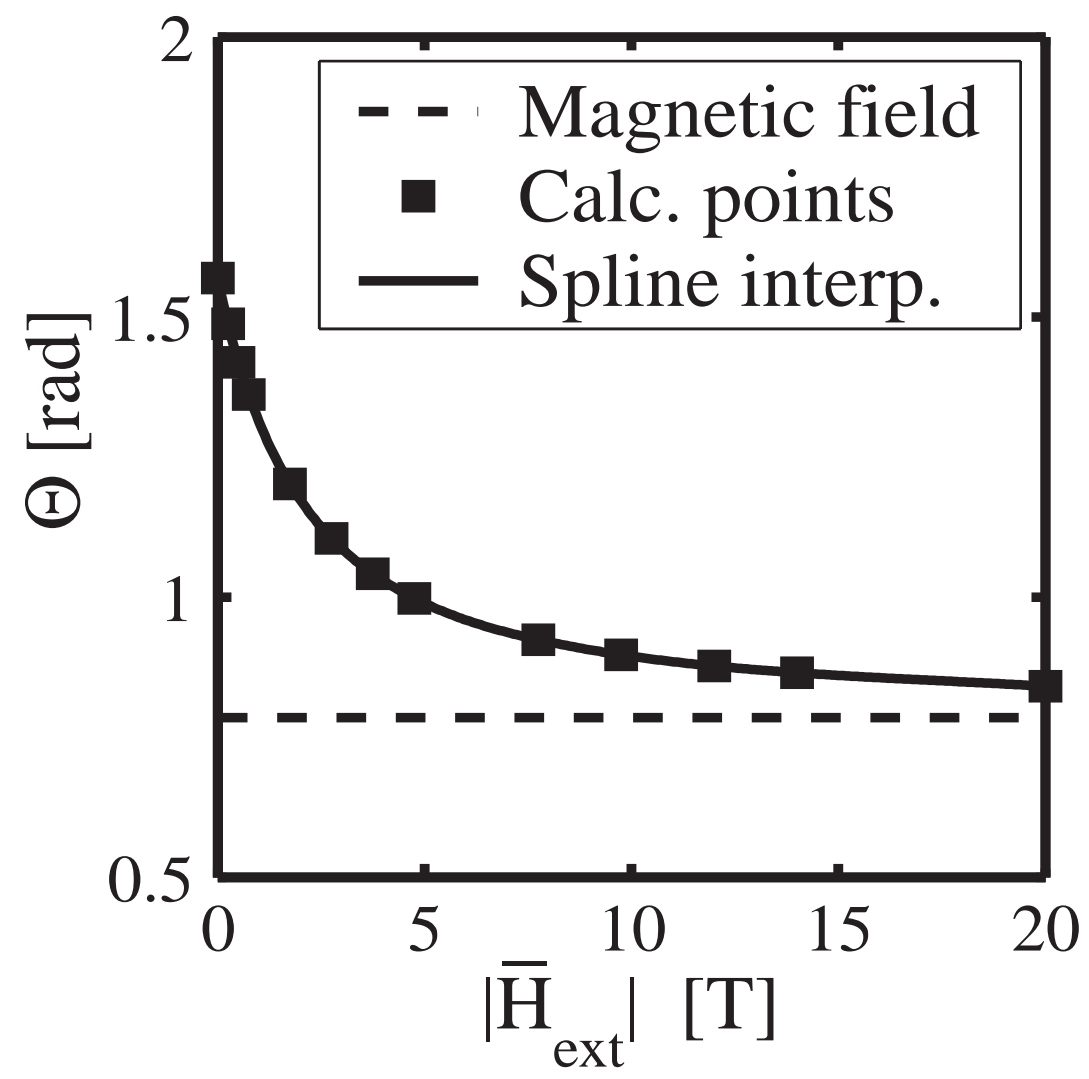

FIG. 15: Variation of the magnetization direction of the stable minimum of Fig. [14], as a function of $\left|\vec{H}_{\text {ext }}\right| . \Theta$ is the angle between $\hat{\Omega}$ and $\hat{z}$. $\vec{H}_{\text {ext }}$ is in the $z x$-plane at $\pi / 4$ from the $\hat{z}$-axis.

simple model captures the essence of classical hysteresis in ferromagnetic nanoparticles. Quantum mechanically, for sufficiently weak fields the system can still sit in the quantum state characterized by a magnetic moment pointing along the classically metastable direction until the switching field is reached, the system relaxes to its true ground state, and the magnetization orientation collective coordinate changes discontinuously 443.

With a further increase of the external field, the magnetization is gradually twisted from the easy $x$-axis toward the direction of the magnetic field, as shown in Fig. [15]. At low fields $\left(\left|\vec{H}_{\text {ext }}\right|<1 \mathrm{~T}\right)$, the direction of the magnetization corresponding to this local minimum is very close to the $x y$-plane.

Because our hemispherical nanoparticles have a nearly isotropic easy $x y$-plane, the magnetization will rotate in the $x y$-plane in response to weak applied external fields 44 This is exemplified in Fig. [16], where we plot the the total energy as a function of $\left|\vec{H}_{\text {ext }}\right|$, when $\Omega$ lies in the $x y$-plane. $\left|\vec{H}_{\text {ext }}\right|$ is oriented in the direction $(\theta=\pi / 4, \phi=\pi / 16)$. In the absence 


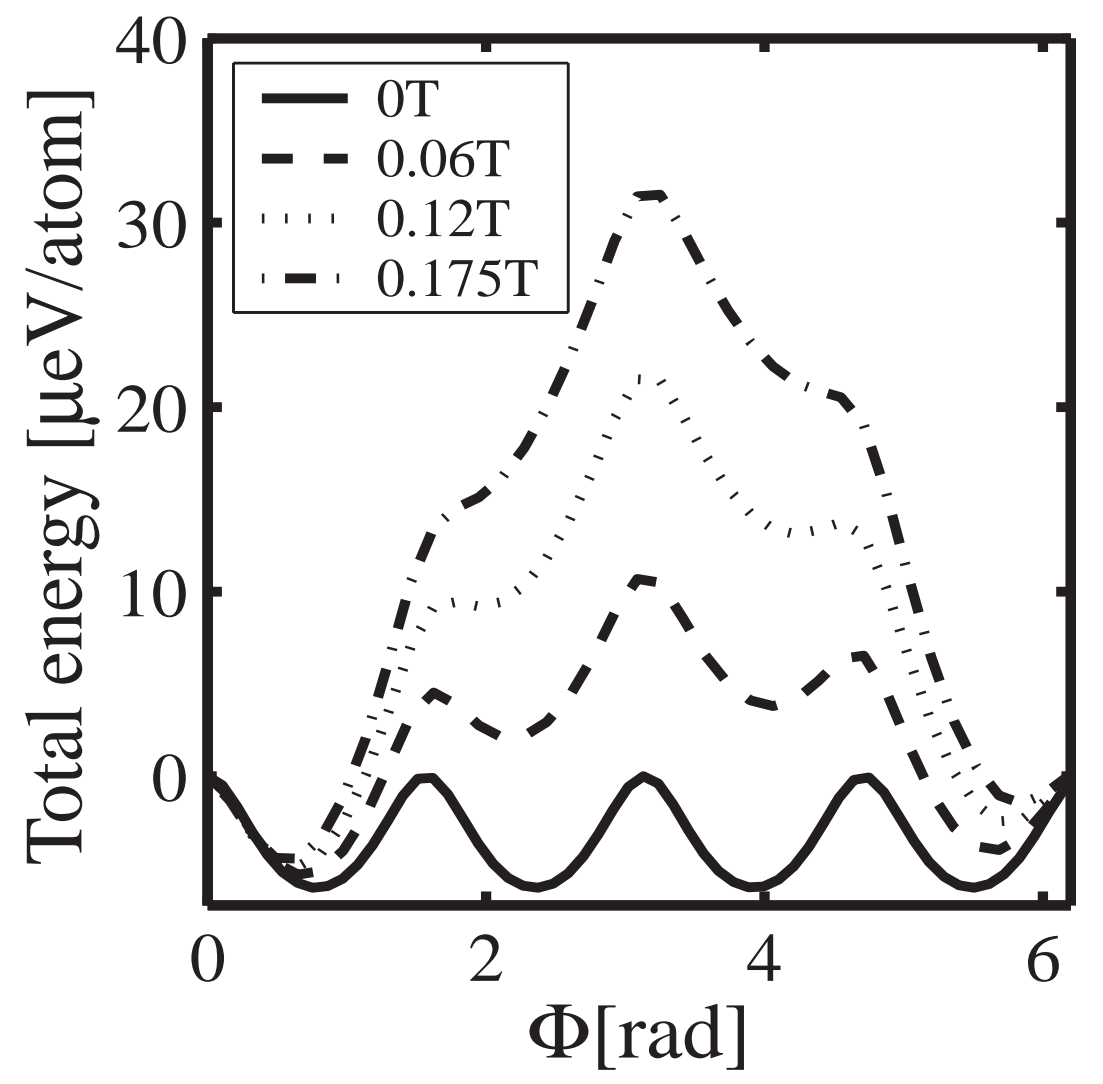

FIG. 16: Anisotropy energy in the $x y$-plane for different magnitudes of an external magnetic field. The direction of the external field is $(\theta=\pi / 4, \phi=\pi / 16)$. $\Phi$ is the angle between $\hat{\Omega}$ (lying in the $x y$-plane) and $\hat{x}$.

of the external field, the ground state of the nanoparticle is four-fold degenerate, with the degeneracy corresponding to one of the four magnetization directions $\pm \hat{x} \pm \hat{y}$, associated to the four local minima of Fig. [16]. As the external field increases, three of these minima will become classically metastable with small barriers separating them from each other and the true ground state. In general the different local minima will lose their metastability at different external field strengths. In the case we consider, the first switching field is reached at $\left|\vec{H}_{\text {ext }}\right| \approx 0.15 \mathrm{~T}$, when the minimum originally at $-\hat{x}-\hat{y}$ disappears. If the system starts out in this local minimum at zero external field, at the switching field it will jump to the ground state with a corresponding rotation of the magnetization in the $\hat{x}+\hat{y}$-direction. Since everything takes place essentially within the $x y$-plane, where the typical anisotropy energies are one order of magnitude smaller than in the $z x$-plane, the scale of the coercivity is also much smaller than the one in the $z x$-plane. More complicated hysteretic behaviors are also 
possible, in which, by starting from a different minimum at zero external field, the system jumps first from one metastable to another metastable state and only at a second switching field reaches its true ground state.

\section{B. Dependence of quasiparticle levels on external fields}

The hysteretic behavior that we have seen in the ground-state properties of a ferromagnetic nanoparticle has profound implications for the magnetic field dependence of its low-energy elementary excitations. In ferromagnetic metals there exist two kinds of elementary excitations: collective spin excitations associated with magnetization orientation degrees of freedom, and particle-hole excitations. In a ferromagnetic nanoparticle the distinction between these two kinds of excitations is partly obscured by the effect of spinorbit coupling[11, 36]. Sorting out this problem is particularly important in order to interpret current tunneling experiments in single-electron transistors [7]. Here we examine only particle-hole excitations around the Fermi level, which are immediately available within our Hartree-Fock treatment of the many-body Hamiltonian. In Fig. [17] we plot the magnetic field dependence of a few single-particle energy levels around the Fermi energy. As a simplified illustration, the levels are calculated assuming that the ground-state dependence on the external field is as described in Fig. [14]: at low field the ground-state magnetization is oriented around the $-\hat{x}$-axis until the switching field is reached, whereupon the magnetization is reversed along a direction around the $\hat{x}$-axis. The corresponding field dependence of the quasiparticle is quite complex. In the small-field regime $\left(\left|\vec{H}_{\text {ext }}\right|<1 \mathrm{~T}\right)$ there is a hysteretic switching at a certain $\left|\vec{H}_{\text {ext }}\right|=H_{\mathrm{sw}} \approx 1 \mathrm{~T}$, due to an abrupt change of the ground-state magnetic moment. There is basically no correlation between single particle states before and after reversal. Notice in particular that the levels can jump either up or down at $H_{\mathrm{sw}} 45$. Furthermore the quasiparticle energies have continuous non-monotonic variations, which seem to differ randomly from level to level. In the large field regime $\left(\left|\vec{H}_{\text {ext }}\right|>>H_{\text {sw }}\right)$, the quasiparticle energies depend roughly linearly on $\left|\vec{H}_{\text {ext }}\right|$ and their slopes almost all have the same sign. In the small-field behavior, the variation of the quasiparticle energies as a function of the external field is due to a combination of the rotation of the particle's magnetic moment direction $\Omega$, and Zeeman coupling. We have shown above (see Fig. [3]) that the dependence of the quasiparticle energies on $\hat{\Omega}$ varies randomly from level to level. Thus the 

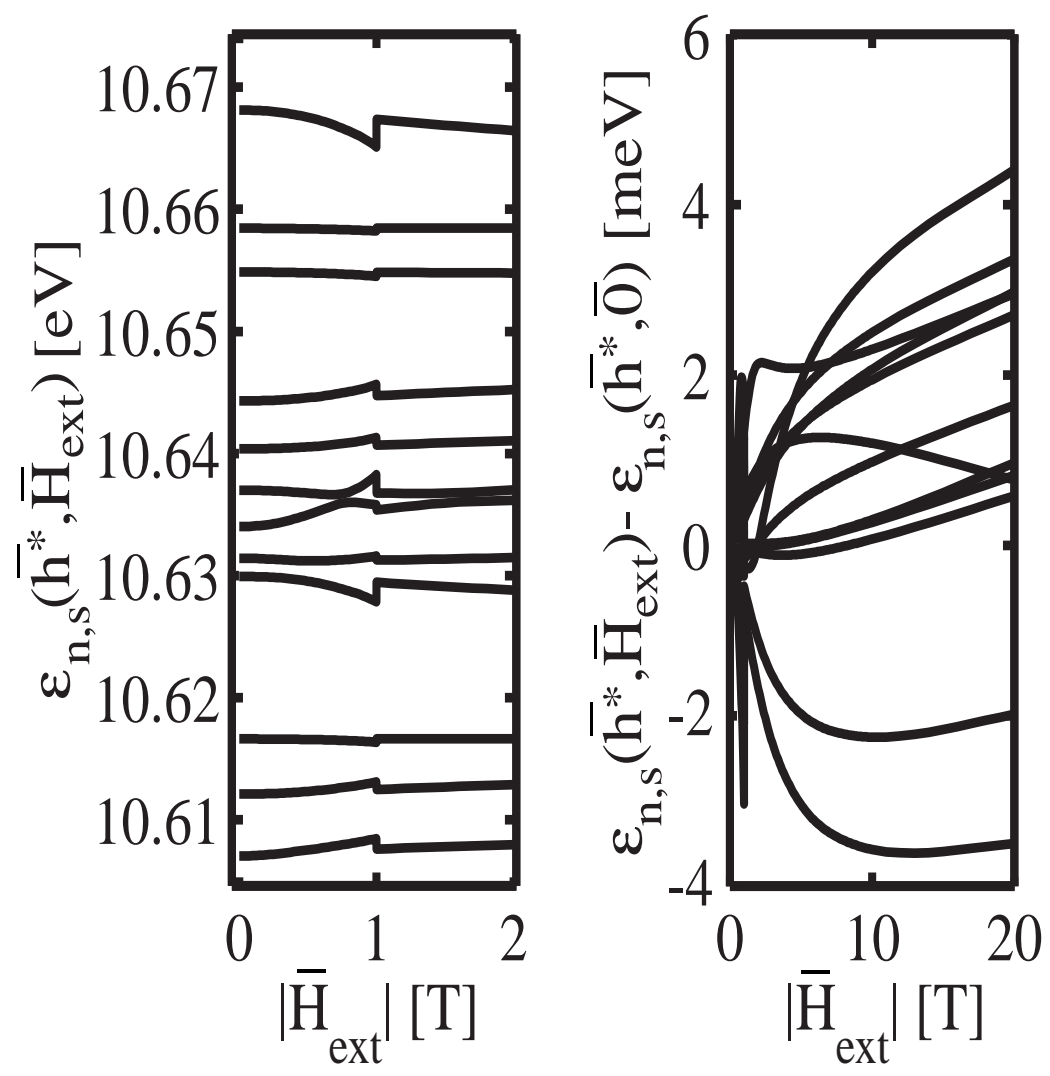

FIG. 17: Variation of quasiparticle energy levels $\epsilon_{n, s}\left(\vec{h}^{\star}, \vec{H}_{\text {ext }}\right)$ around the Fermi level in an external magnetic field. The levels are calculated assuming that the ground-state of the nanoparticle changes in the external field in the way described in Fig. [14].

complex non-monotonic behavior at small fields can be understood within our model. At large fields $\left(\left|\vec{H}_{\text {ext }}\right|>>H_{\mathrm{sw}}\right) \hat{\Omega}$ is slowly twisted toward the field direction and the Zeeman coupling plays the dominating role in the field dependence. Moreover, because the Fermi level lies in a region of predominantly minority-spin energy levels, it is expected that almost all particle and hole excitations around the Fermi level have the same high-field slope[8].

In the discussion above we have assumed, out of simplicity, that the magnetization is arbitrarily constrained to rotate in the $z x$-plane. At weak external fields, however, the magnetization will stay close to the $x y$-plane. For a weak external field in the $(\theta=\pi / 4, \phi=$ $\pi / 16)$ direction, the actual dependence of the ground-state energy on the magnetization direction at different field strengths will be the one represented in Fig. [16]. Consequently, the change of quasi-particle energies as a function of the external field will reflect this more complicated behavior. We illustrate this point in Fig. [18-a] where, as an example, we 

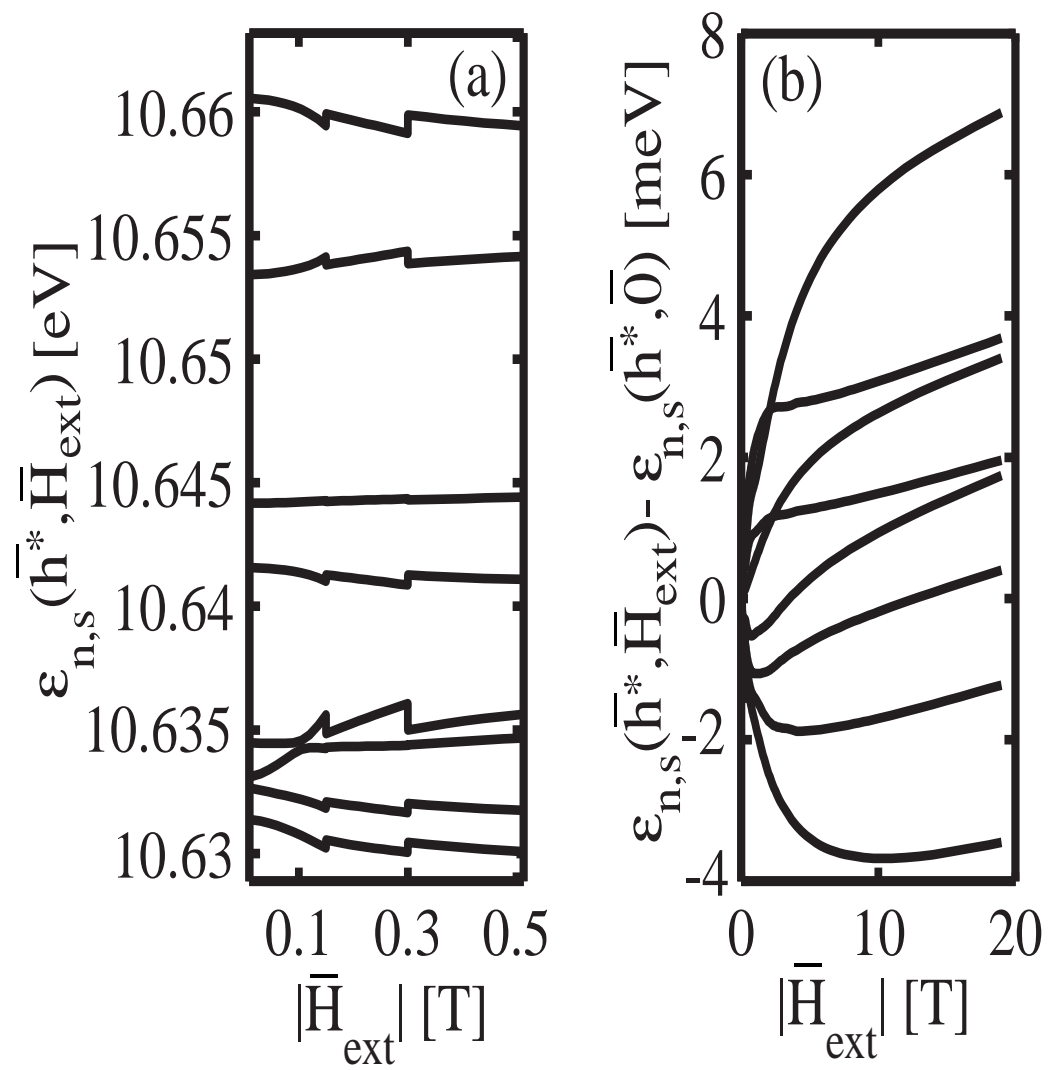

FIG. 18: Variation of quasiparticle energy levels $\epsilon_{n, s}\left(\vec{h}^{\star}, \vec{H}_{\text {ext }}\right)$ around the Fermi level in an external magnetic field. (a) The levels are calculated assuming that the ground-state of the nanoparticle changes in the external field in the way described in Fig. [16]. (b) For magnetic fields larger than 1 $\mathrm{T}$, the magnetization starts to develop a non-negligible component perpendicular to the $x y$ plane, while its projection in the $x y$-plane is parallel to the component of $\vec{H}_{\text {ext }}$ in that plane.

assume that at zero field the magnetization points in the $-\hat{x}+\hat{y}$ direction. As the magnetic field increases, there will be a first jump in the in the quasiparticle-level dependence at $H_{\mathrm{sw}} \approx 0.15 \mathrm{~T}$, when the magnetization re-orients itself in the $(+\hat{x}+\hat{y})$-direction. There will a second hysteresis jump in the quasi-particle energies at $H_{\mathrm{sw}} \approx 0.3 \mathrm{~T}$, when the magnetic moment finally switches to the $(+\hat{x}-\hat{y})$-direction, corresponding to the only metastable configuration left at this field. For a different starting local minimum at $\left|\vec{H}_{\text {ext }}\right|=0$, the variation of the quasiparticle energies as a function of the external field will be in general different. By increasing the magnetic field beyond $1 \mathrm{~T}$, the magnetization will start to develop a non negligible component perpendicular to the $x y$-plane, while its component in the $x y$-plane will become essentially frozen along the direction of the component of $\vec{H}_{\text {ext }}$ in 
that plane.

The quasiparticle-energy dependence on an external magnetic field presented here, has striking similarities with the field dependence of the tunneling resonance energies in singleelectron tunneling experiments [1, [7]. Notice that for the hysteretic behavior occurring as a result of the rotation of the magnetization in the vicinity of the easy $x y$-plane, the order

of magnitude of the coercivity that we find is close to the experimental value. The only discrepancy between the results of our theoretical model and the experiment is the mean level spacing $\delta_{\text {res }}$ of the low-energy excitations. Since in our model we are only taking into account Hartree-Fock quasiparticles, the expected level spacing of the low-lying excitation is approximately $\delta_{\downarrow}$, which is a few meV in our 143-atom nanoparticle and should be of the order of $0.5 \mathrm{meV}$ for a 1500 -atom nanoparticle considered in the experiment.

This value is still larger than the value $\delta_{\text {res }} \leq 0.2 \mathrm{meV}$ observed experimentally. We believe that a unified and consistent inclusion of collective spin excitations, and possibly also the non-equilibrium transport effects proposed in Refs. [9, 10], could resolve this confusion.

\section{CONCLUSIONS}

In this article we have investigated the effects of spin-orbit interactions on the properties of ferromagnetic metal nanoparticles. In particular, we have focused on their novel microscopic magnetocrystalline anisotropy physics, and on hysteresis in the quasiparticle excitations spectra of metallic nanomagnets. Our analysis, based on qualitative considerations backed up by numerical studies of a generic model for ferromagnetic transition metal nanoparticles, provides an understanding of some emergent properties of their quasiparticle states. We find two regimes separated by a broad crossover and characterized by the comparison of several characteristic energy scales. For small nanoparticles with fewer than $\mathcal{N}_{a} \sim 200$ atoms, the single particle mean-level spacing $\delta$ is larger than spin-orbit induced energy-shifts $\epsilon_{\mathrm{SO}}$ in the quasi-particle spectra. These shifts have the same typical size as the spin-orbit scattering lifetime broadening energies of very large particles, $\hbar \tau_{\mathrm{SO}}^{-1}$. The quasi-particle levels of small nanoparticles in which $\delta \tau_{\mathrm{SO}}>\hbar$ evolve in a complicated way as a function of magnetization orientation and external magnetic field, with relatively small avoided crossing gaps and spin-orbit shifts of nearby orbitals that are nearly uncorrelated. The size of the avoided crossing gaps is determined by matrix element of the spin-orbit 
coupling operator between quasiparticle energy levels that are adjacent in energy. Surprisingly, even though expectation values of these matrix elements vanish because of angular momentum quenching, typical values between energetically adjacent orbitals are comparable to those for orbitals at any position in the spectrum. Eventually, for nanoparticles with more than $\sim 1000$ atoms, the typical avoided crossing gap estimated in this way becomes comparable to the level spacing and we expect the crossover to the strong coupling limit is complete. Nanoparticles with fewer than $\mathcal{N}_{a} \sim 1000$ atoms can easily be prepared with current synthesis techniques and systems of experimental interest are often in the middle of the crossover between small particle and bulk (weak and strong spin-orbit coupling) limits. For example, the nanoparticles investigated by electron tunneling experiments contain between 50 and 1500 atoms [1, [].

For nanoparticles in the size range we are able to study numerically, $\mathcal{N}_{a}$ smaller than a few hundred, we find that the anisotropy energy per atom displays large changes of order several percent when the electron or atom number changes by one. Our analysis allows us to make a connection between the microscopic model of a metal nanomagnet and more familiar classical micromagnetic energy functional expressions which are normally used to interpret the results of switching-field experiments[3]. The ground-state energy as a function of the magnetization direction is characterized by minima separated by energy barriers. The quasi-particle levels exhibit a complex non-monotonic behavior and abrupt jumps when the magnetization direction is reversed by an external magnetic field. The nanoparticles investigated by electron tunneling experiments contain between 50 and 1500 atoms [1, [7]. The results that we have presented here are therefore particularly relevant for the interpretation of these experiments. In particular, we find that the anisotropy fluctuations inferred from interpretations of these experiments are indeed to be expected. Similarly, the dependence of the tunneling resonances on the external magnetic field is qualitatively similar to the behavior of the quasi-particle excitations of our model, although it appears necessary to invoke non-equilibrium quasiparticle configurations 10 in the nanoparticle in order to understand the size of the quasiparticle level spacing. 


\section{ACKNOWLEDGEMENTS}

It is a pleasure to thank Mandar Deshmukh and Dan Ralph for several discussions about their experimental results, and Jan von Delft for stimulating and informative interactions. C.M.C. would like to acknowledge helpful discussions with L. Samuelson and with the participants of the 2001 workshop "Spins in Nanostructures", at the Aspen Center for Physics, where part of this work was completed. A.H.M. acknowledges informative discussions with W. Wernsdorfer and P. Brouwer. This work was supported in part by the Swedish Research Council under Grant No:621-2001-2357 and in part by the National Science Foundation under Grant DMR 0115947.

[1] S. Guéron, M. M. Deshmukh, E. B. Myers, and D. C. Ralph, Phys. Rev. Lett. 83(20), 4148 (1999).

[2] C. T. Black, C. B. Murray, R. L. Sandstrom, and S. Sun, Science 287(5460), 1989 (2000).

[3] M. Jamet, W. Wernsdorfer, C. Thirion, D. Mailly, V. Dupuis, P. Mélinon, and A. Péres, Phys. Rev. Lett. 86, 4676 (2001).

[4] E. C. Stoner and E. P. Wohlfart, Phil. Trans. Phil. Roy. Soc. (London) 240, 599 (1948).

[5] H. Zijlstra, in Ferromagnetic Materials, edited by E. Wohlfarth (North-Holland, New York, 1982), vol. 3, p. 37.

[6] R. Skomski and J. M. D. Coey, Permanent Magnetism (Institute of Physics, Bristol, 1999).

[7] M. M. Deshmukh, S. G. S. Kelff, E. Bonnet, A. N. Pasupathy, J. von Delft, and D. C. Ralph, Phys. Rev. Lett. 87, 226801 (2001).

[8] C. M. Canali and A. H. MacDonald, Phys. Rev. Lett. 85(26), 5623 (2000).

[9] S. Kelff, J. von Delft, M. M. Deshmukh, and D. C. Ralph, Phys. Rev. B 64, 220401 (2001).

[10] S. Kleff and J. von Delft, Nonequilibrium excitations in ferromagnetic nanoparticles (2001), unpublished.

[11] A. H. MacDonald and C. M. Canali, Solid State Comm. 119, 253 (2001).

[12] J. C. Slater and G. F. Koster, Phys. Rev. 94(6), 1498 (1954).

[13] L. F. Mattheiss, Phys. Rev. B 2(10), 3918 (1970).

$[14]$ D. A. Papaconstantopoulos, Handbook of the Band Structure of Elemental Solids (Plenum, 
New York, 1986).

[15] H. M. Duan and Q. Q. Zheng, Phys. Lett. A 280(5), 333 (2001).

[16] I. M. L. Billas, A. Châtelain, and W. A. de Heer, Science 265, 1682 (1994).

[17] I. M. L. Billas, A. Cht̂telain, and W. A. D. Heer, J. Magn. Magn. Mater. 168(1), 64 (1997).

[18] P. Bruno, Phys. Rev. B 39(1), 865 (1989).

[19] H. Takayama, K. P. Bohnem, and P. Fulde, Phys. Rev. B 14(6), 2287 (1976).

[20] D. D. Koelling and A. H. MacDonald, in Relativistic Effects in Atoms, Molecules, and Solids, edited by E. E. Malli (Plenum Press, New York, 1983), vol. 87 of NATO Advanced Study Institute, Ser. B, p. 227.

[21] J. Dorantes-Dávila and G. M. Pastor, Phys. Rev. Lett. 81(1), 208 (1998).

[22] O. Ericsson, B. Johansson, R. C. Albers, A. M. Boring, and M. S. S. Brooks, Phys. Rev.B 42(4), 2707 (1990).

[23] H. J. Jansen, Physics Today 48(4), 50 (1995).

[24] T. Oda, A. Pasquarello, and R. Car, Phys. Rev. Lett. 80(16), 3622 (1998).

[25] A. Cehovin, Nonhomogenous self-consistent hartee-fock approximation in ferromagnetic metal nanoparticles (2001), unpublished.

[26] W. Wernsdorfer, E. B. Orozco, A. Benoit, D. Mailly, O. Kubo, H. Nakamo, and B. Barbara, Phys. Rev. Lett. 79(20), 4014 (1997).

[27] G. M. Pastor, J. Dorantes-Dávila, S. Pick, and H. Dreyssé, Phys. Rev. Lett. 75(20), 326 (1995).

[28] P. Bruno and J. Seiden, J. Phys. C 49(8), 1645 (1988).

[29] P. Bruno, G. Bayreuther, and P. Beauvillain, J. Appl. Phys. 68(11), 5759 (1990).

[30] M. B. Stearns, in 3d, 4d and 5d Elements, Alloys and Compounds, edited by H. Wijn and N. S. Landolt-Boerstein (Springer, Berlin, 1986), vol. 19, Group III, Pt. a, p. 34.

[31] W. P. Halperin, Rev. Mod. Phys. 58, 533 (1986).

[32] P. W. Brouwer, X. Waintal, and B. I. Halperin, Phys. Rev. Lett 85, 369 (2000).

[33] K. A. Matveev, L. I. Glazman, and A. I. Lakin, Phys. Rev. Lett 85, 2789 (2000).

[34] J. R. Petta and D. C. Ralph, Phys. Rev. Lett 86, 266801 (2001).

[35] M. D. Stiles, S. V. Halilov, R. A. Hyman, and A. Zangwill, Phys. Rev. B 65(10), 104430 (2001).

[36] C. M. Canali, A. Cehovin, and A. H. MacDonald, Collective excitations in ferromagnetic metal 
nanoparticles (2001), unpublished.

[37] The choice of a hemisphere is motivated by the tunneling experiments of Ref. [1, 7]. The crystal structure could not be identified in these experiments. High resolution transmission electron microscopy measurements on Co clusters prepared in a different way[3] have shown that the nanoparticles are well crystallized in the f.c.c. structure even though bulk Co has an h.c.p. structure.

[38] Notice however, that the one body term, $H_{\text {band }}$ implicitly includes a mean-field approximation to those portions of the interaction not captured by the exchange term.

[39] More precisely, this is the magneto-electric contribution to the magneto-crystalline anisotropy.

[40] The additional anisotropy due to magnetic dipole interactions is also sensitive to the overall nanoparticle shape, but is not included here because it is generally less important for nanoparticles than for macroscopic samples. This contribution to the anisotropy is additive to a good approximation, does not have substantial mesoscopic fluctuations, and can be simply added to the effects discussed here when it is important.

[41] The use of two Fermi energies for majority and minority spins can generate some confusion: in this terminology, the energies are pure kinetic (or band) energies, i.e the exchange contribution is not included. When the exchange energy is included, as we do below in the context of meanfield theory, majority and minority spins have the same Fermi level.

[42] This is approximately the value of the single-particle mean-level at the Fermi level also when spin-orbit interaction is included.

[43] This scenario neglects the possibility of quantum tunneling between the two local minima through the energy barrier separating them. At low fields and for nanoparticles of 1-4 nanometers in diameter, quantum tunneling is exponentially small.

[44] Clearly, if the external field is not in the $x y$-plane the magnetization will not stay exactly in this easy plane. However the displacement of the magnetization from easy to hard directions is negligible at the weak fields $\left(\left|\vec{H}_{\text {ext }}\right|<1 T\right)$ (See Fig. [15] ) and it becomes relevant only at larger fields, when the component of the magnetization in the $x y$-plane is essentially frozen along the direction of the component of $\left|\vec{H}_{\text {ext }}\right|$ in that plane.

[45] The quasiparticle energy difference on the opposite sides of the transition is of the order of the typical quasiparticle anisotropy energy. For the nanoparticles that we can study numerically this energy is smaller than the mean level spacing. However for larger particles the energy 
difference at the transition can be larger than $\delta$. In this case the jumps at the switching fields will cause a complete reshuffling of the quasiparticle levels. 